\title{
Adiabatic Eigenstate Deformations as a Sensitive Probe for Quantum Chaos
}

\author{
Mohit Pandey, ${ }^{1}$ Pieter W. Claeys $\odot,{ }^{1,2}$ David K. Campbell@ ${ }^{1}$ Anatoli Polkovnikov, ${ }^{1}$ and Dries Sels ${ }^{3,4, *}$ \\ ${ }^{1}$ Department of Physics, Boston University, 590 Commonwealth Avenue, Boston, \\ Massachusetts 02215, USA \\ ${ }^{2}$ TCM Group, Cavendish Laboratory, University of Cambridge, Cambridge CB3 OHE, United Kingdom \\ ${ }^{3}$ Department of Physics, Harvard University, Cambridge, Massachusetts 02138, USA \\ ${ }^{4}$ Theory of quantum and complex systems, Universiteit Antwerpen, B-2610 Antwerpen, Belgium
}

(Received 10 April 2020; accepted 14 September 2020; published 23 October 2020)

\begin{abstract}
In the past decades, it was recognized that quantum chaos, which is essential for the emergence of statistical mechanics and thermodynamics, manifests itself in the effective description of the eigenstates of chaotic Hamiltonians through random matrix ensembles and the eigenstate thermalization hypothesis. Standard measures of chaos in quantum many-body systems are level statistics and the spectral form factor. In this work, we show that the norm of the adiabatic gauge potential, the generator of adiabatic deformations between eigenstates, serves as a much more sensitive measure of quantum chaos. We are able to detect transitions from integrable to chaotic behavior at perturbation strengths orders of magnitude smaller than those required for standard measures. Using this alternative probe in two generic classes of spin chains, we show that the chaotic threshold decreases exponentially with system size and that one can immediately detect integrability-breaking (chaotic) perturbations by analyzing infinitesimal perturbations even at the integrable point. In some cases, small integrability breaking is shown to lead to anomalously slow relaxation of the system, exponentially long in system size.
\end{abstract}

DOI: $10.1103 /$ PhysRevX.10.041017

\section{INTRODUCTION}

Finding signatures of chaos in the quantum world has been a long-standing puzzle [1-3]. In the past few years, exciting progress has been made on characterizing the effects of chaos on dynamical properties of quantum manybody systems; see Fig. 1 [4-11]. Classical chaos is usually described in terms of an exponential sensitivity of trajectories to initial conditions [12]. However, the quantum world precludes any definition of chaos in terms of physical trajectories due to the Heisenberg uncertainty principle. Alternatively, chaos can be defined in terms of the absence of integrability. Classical Liouville-Arnold integrability is formulated in terms of independent Poisson-commuting integrals of motion. Again, although there have been many attempts to characterize quantum integrability in a similar way, no such unique definition exists [13-16].

In the past two decades, random matrix theory (RMT) [17-19] has shown outstanding success in the understanding of quantum chaos. Following the work of Wigner [20,21],

\footnotetext{
*dsels@nyu.edu
}

Published by the American Physical Society under the terms of the Creative Commons Attribution 4.0 International license. Further distribution of this work must maintain attribution to the author(s) and the published article's title, journal citation, and DOI.
Subject Areas: Complex Systems, Quantum Physics, Statistical Physics
Bohigas, Giannoni, and Schmit [22] conjecture that the energy-level statistics of all quantum systems whose classical analogs are chaotic should show level repulsion and belong to one of three universal classes depending upon their symmetry: the Gaussian orthogonal ensemble, the Gaussian unitary ensemble, or the Gaussian symplectic ensemble. On the other hand, according to the Berry-Tabor conjecture 23]], integrable systems have uncorrelated energy levels and usually exhibit Poissonian level spacing statistics. These ideas were later extended to generic quantum systems and tested numerically under the general framework of the eigenstate thermalization hypothesis (ETH) [24-29]. By now, the emergence of the random matrix behavior of quantum eigenstates is an accepted definition of quantum chaos.

Numerically, two additional steps are required before one can accurately compare the statistical properties (e.g., through level statistics or the spectral form factor [30,31]) of a particular quantum system with the predictions of RMT: (i) remove any symmetries and (ii) rescale the spectrum, setting the local mean level spacing to unity (also called unfolding the spectrum). First, if symmetries are not removed, energy levels in different symmetry sectors do not have any correlations, so that spectra of chaotic systems can show Poissonian distributions [32,33]. However, finding all symmetries of a many-body Hamiltonian is computationally hard without any physical intuition, since this task effectively involves searching for 
all possible (local) operators that commute with the Hamiltonian. Second, there are various methods to unfold the spectrum, and it is known that statistics, especially ones measuring long-range correlations, can be sensitive to the adopted unfolding procedure [34]. Moreover, the procedure can also exhibit finite-size effects. In light of these issues, it is advisable rather to use the ratio of two consecutive level spacings [35,36] or survival probability (see Refs. [37,38]).

Here, we propose an alternative tool to detect chaos in quantum systems, based on the rate of deformations of eigenstates under infinitesimal perturbations. Mathematically, the distance between nearby eigenstates (also known as the Fubini-Study metric [39-42]) can be expressed as the Frobenius norm of the so-called adiabatic gauge potential (AGP) [39,43-45], which is exactly the operator that generates such deformations. It is straightforward to show that this norm should scale exponentially with the system size in chaotic systems satisfying the ETH [39]. In this sense, quantum chaos manifests itself through an exponential sensitivity of the eigenstates to infinitesimal perturbations, which can be viewed as an analog to classical chaos, reflected in the exponential sensitivity of trajectories to such perturbations. Moreover, unlike standard probes of RMT such as the spectral form factor (see, e.g., Ref. [46]) or the closely related survival probability (see Refs. [37,38]), as well as level statistics, which depend only on the eigenvalues of the Hamiltonian, the AGP norm is sensitive to both the level spacings and the specific kind of adiabatic deformation (perturbation).

We find that the norm of the AGP shows a remarkably different, and extremely sensitive, scaling with system size for integrable and chaotic systems: polynomial versus exponential. In our method, we do not need to remove any symmetries before computing the AGP norm needed in the analysis of the level spacing distributions and do not need to average over different Hamiltonians, which is necessary to analyze the (non-self-averaging) spectral form factor. We show that one can detect chaos through the sharp crossover between the polynomial and exponential scaling of the norm. The sensitivity of this norm to chaotic perturbations is orders of magnitude greater than that of the aforementioned methods. Using this approach, we find several, previously unexpected, results for a particular but fairly generic integrable $X X Z$ spin chain with additional small perturbations: (i) The strength of the integrabilitybreaking perturbation scales exponentially down with the system size, much faster than in previous estimates [47,48]; (ii) integrability-breaking deformations immediately lead to an exponential scaling of the norm of the AGP, showing that chaotic perturbations can be already detected in the integrable regimes; and (iii) in the presence of small integrability-breaking terms, the system can exhibit exponentially slow relaxation dynamics, which is similar to the slow dynamics observed in some classical nearly integrable systems like the Fermi-Pasta-Ulam-Tsingou (FPUT) chain [49-51]. We also find that such relaxation dynamics are

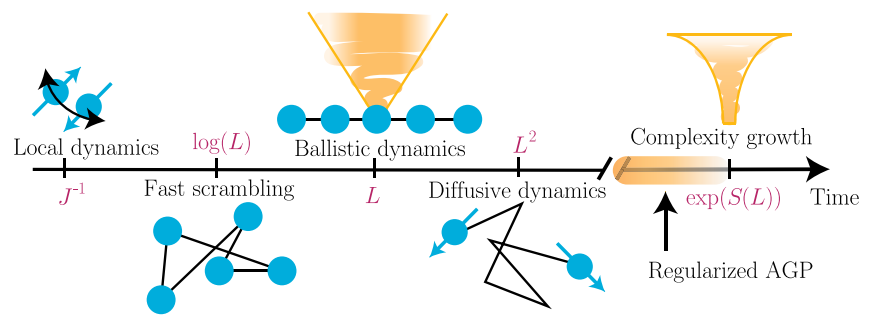

FIG. 1. Signatures of chaos. Quantum chaos manifests itself in a vast range of different phenomena, each relevant up to a particular system-size-dependent timescale. At the earliest times, where dynamics are limited by the local bandwidth, one can see the onset of chaos. In systems without spatial locality, this limit could lead to fast scrambling, allowing one to identify a Lyapunov exponent. Systems with spatial locality are further characterized by an additional, so-called, butterfly velocity. While this ballistic propagation ends at times $O(L)$, diffusive dynamics continues up to the Thouless time $O\left(L^{2}\right)$. All local dynamics has now come to a stop; nonetheless, operators keep spreading over operator space, becoming increasingly more complex. This process continues for exponentially long times, stopping only at the Heisenberg time $\exp [S(L)]$.

very different for observables conjugate [see Eq. (2) below] to integrable and chaotic directions (perturbations) of the Hamiltonian. We find similar results for an Ising model, where the integrability is broken by introducing a longitudinal field.

The connection with relaxation is not surprising, since one representation of the AGP is in terms of the long-time evolution of a local operator conjugate to the coupling. Hence, our results relate to recent studies of information propagation through operator growth in quantum manybody systems [52-54], where chaotic and integrable systems are again expected to exhibit qualitatively different behavior (e.g., in operator entanglement $[55,56]$ and Lanczos coefficients $[57,58])$. Whereas most of the previous works focus mainly on short-time effects, here we effectively focus on dynamics and operator growth at times that are exponentially long in the system size (Fig. 1).

\section{ADIABATIC GAUGE POTENTIAL}

Before proceeding, let us define the AGP and discuss some of its key properties. Given a Hamiltonian $H(\lambda)$ depending on a parameter $\lambda$, the adiabatic evolution of its eigenstates as we vary this parameter is generated by the AGP as (in units with $\hbar=1$ )

$\mathcal{A}_{\lambda}|n(\lambda)\rangle=i \partial_{\lambda}|n(\lambda)\rangle, \quad H(\lambda)|n(\lambda)\rangle=E_{n}(\lambda)|n(\lambda)\rangle$.

Using the Hellmann-Feynman theorem, it is easy to see that the matrix elements of the AGP between such eigenstates are given by

$$
\left\langle m\left|\mathcal{A}_{\lambda}\right| n\right\rangle=-\frac{i}{\omega_{m n}}\left\langle m\left|\partial_{\lambda} H\right| n\right\rangle,
$$


where $\omega_{m n}=E_{m}(\lambda)-E_{n}(\lambda), \partial_{\lambda} H$ is the operator conjugate to the coupling $\lambda$, and we make the dependence on $\lambda$ implicit. The diagonal elements of $\mathcal{A}_{\lambda}$ can be chosen arbitrarily due to the gauge freedom in defining the phases of eigenstates. A convenient choice consists of setting all diagonal elements equal to zero. For simplicity, we assume there are no degeneracies in the spectrum, but, as will be clear shortly, this assumption is not necessary and does not affect any of the results below. We define the $L_{2}$ (Frobenius) norm, also called the Hilbert-Schmidt norm, of this operator as

$$
\left\|\mathcal{A}_{\lambda}\right\|^{2}=\frac{1}{\mathcal{D}} \sum_{n} \sum_{m \neq n}\left|\left\langle n\left|\mathcal{A}_{\lambda}\right| m\right\rangle\right|^{2},
$$

where $\mathcal{D}$ is the dimension of the Hilbert space.

This expression should scale exponentially with the system size in chaotic systems satisfying the ETH: $\left\|\mathcal{A}_{\lambda}\right\|^{2} \sim \exp [S]$, where $S$ is the entropy of the system [39]. Within the ETH, the off-diagonal matrix elements of local operators, including $\partial_{\lambda} H$, scale as $\left\langle m\left|\partial_{\lambda} H\right| n\right\rangle \propto$ $\exp [-S / 2][25,28]$, while the minimum energy gap between states, $\omega_{m n}$, scales as $\exp [-S]$. The scaling of individual matrix elements is already explored in the literature to study the crossover between chaotic and nonergodic behavior, e.g., in the context of disordered systems [59,60] and integrability breaking [61,62]; see the Appendix G. As we demonstrate, the exponential scaling of the norm of the AGP can be used to detect the emergence of chaotic behavior in the system with tremendous (exponential) precision.

However, Eq. (2) is not particularly convenient: The norm of the exact AGP can be dominated by the smallest energy difference between eigenstates, and, as such, it is highly unstable and difficult to analyze, especially close to the ergodicity transition. Accidental degeneracies in the spectrum that are lifted by $\partial_{\lambda} H$ also cause the norm to formally be infinite. To resolve this issue, it is convenient to instead define a "regularized" AGP as follows:

$$
\left\langle m\left|\mathcal{A}_{\lambda}(\mu)\right| n\right\rangle=-i \frac{\omega_{m n}}{\omega_{m n}^{2}+\mu^{2}}\left\langle m\left|\partial_{\lambda} H\right| n\right\rangle,
$$

where $\mu$ is a small energy cutoff. For the sake of brevity, we drop the argument $\mu$, and, unless specified otherwise, $\mathcal{A}_{\lambda}$ refers to the regularized AGP. This change has a clear physical intuition: Instead of considering transitions (matrix elements) between individual eigenstates, we now consider only transitions between energy shells with width $\mu$. For eigenstates with $\left|\omega_{m n}\right| \gg \mu$, this process reproduces the exact AGP, whereas in the limit $\left|\omega_{m n}\right| \ll \mu$, the AGP no longer diverges but reduces to a constant. Alternatively, within the operator growth representation [see Eq. (10) below], $\mu^{-1}$ has the interpretation of a cutoff time. Numerically, this regularization has the immediate advantage that it gets rid of any problem with (near-)divergences. Note that $\mu$ does not need to be system-size independent for this regularization. Interestingly, as long as $\mu \propto \exp [-S]$, the norm of the AGP within chaotic systems should also remain proportional to $\exp [S]$. We can use this flexibility in defining $\mu$ to our advantage, choosing it to be parametrically larger than the level spacing to eliminate any effect of accidental degeneracies but still exponentially small to minimize the deviation from the exact AGP. We find that choosing $\mu(L) \propto L \exp [-S(L)]$, where $L$ is the system size, is the most convenient choice (see Appendix A).

From Eqs. (3) and (4), the norm of the regularized AGP reads

$$
\begin{aligned}
\left\|\mathcal{A}_{\lambda}\right\|^{2} & =\frac{1}{\mathcal{D}} \sum_{n} \sum_{m \neq n} \frac{\omega_{m n}^{2}}{\left(\omega_{n m}^{2}+\mu^{2}\right)^{2}}\left|\left\langle m\left|\partial_{\lambda} H\right| n\right\rangle\right|^{2} \\
& =\int_{-\infty}^{\infty} d \omega \frac{\omega^{2}}{\left(\omega^{2}+\mu^{2}\right)^{2}} \overline{\left|f_{\lambda}(\omega)\right|^{2}}
\end{aligned}
$$

where in the second equation we replace the summation with an integration over the energy difference $\omega_{m n}=$ $E_{m}(\lambda)-E_{n}(\lambda)$ and also define the response function

$$
\begin{aligned}
\overline{\left|f_{\lambda}(\omega)\right|^{2}} & =\frac{1}{\mathcal{D}} \sum_{n} \sum_{m \neq n}\left|\left\langle n\left|\partial_{\lambda} H\right| m\right\rangle\right|^{2} \delta\left(\omega_{n m}-\omega\right) \\
& =\frac{1}{\mathcal{D}} \sum_{n} \int_{-\infty}^{\infty} \frac{d t}{4 \pi} e^{i \omega t}\left\langle n\left|\left\{\partial_{\lambda} H(t), \partial_{\lambda} H(0)\right\}\right| n\right\rangle_{c},
\end{aligned}
$$

where $\{\cdots\}$ stands for the anticommutator and the connected correlation function is defined as $\left\langle n\left|\partial_{\lambda} H(t) \partial_{\lambda} H(0)\right| n\right\rangle_{c}=$ $\left\langle n\left|\partial_{\lambda} H(t) \partial_{\lambda} H(0)\right| n\right\rangle-\left\langle n\left|\partial_{\lambda} H(t)\right| n\right\rangle\left\langle n\left|\partial_{\lambda} H(0)\right| n\right\rangle$. Formally, this function represents an average over eigenstates $n$ of the sum of the squares of the off-diagonal matrix elements $\left|\left\langle n\left|\partial_{\lambda} H\right| m\right\rangle\right|^{2}$ with a fixed energy difference $\omega_{m n}=\omega$, which can also be obtained as the Fourier transform of the nonequal time correlation function of $\partial_{\lambda} H$. Within the ETH ansatz, this function exactly coincides with the (averaged over eigenstates) square of the function $f_{\lambda}(\omega)$ introduced by Srednicki [25], according to

$$
\begin{gathered}
\left\langle m\left|\partial_{\lambda} H\right| n\right\rangle=f_{\lambda}(\omega, \bar{E}) e^{-S(\bar{E}) / 2} \sigma_{m n}, \\
\omega=E_{m}-E_{n}, \quad \bar{E}=\left(E_{n}+E_{m}\right) / 2,
\end{gathered}
$$

with $\sigma_{n m}$ a random variable with zero mean and unit variance. Recently, it was shown that the function $\left|f_{\lambda}(\omega)\right|^{2}$ remains well defined and smooth in generic integrable systems $[61,63,64]$.

Alternatively, it is convenient to rewrite the regularized AGP as a time integral [65-67]: 


$$
\mathcal{A}_{\lambda}=-\frac{1}{2} \int_{-\infty}^{\infty} d t \operatorname{sgn}(t) e^{-\mu|t|}\left(\partial_{\lambda} H\right)(t),
$$

where $\operatorname{sgn}(t)$ is the sign function and

$$
\left(\partial_{\lambda} H\right)(t)=e^{i H t}\left(\partial_{\lambda} H\right) e^{-i H t}
$$

is the operator conjugate to the coupling $\lambda$ in the Heisenberg representation. The exponential factor $\exp [-\mu|t|]$ can be seen as a particular choice of a filter function in the context of quasiadiabatic continuation [68-70]. Notably, Eq. (10) remains valid for classical systems $[65,66]$, and, therefore, the scaling of the AGP norm can be used to detect classical chaos, which we leave for future work.

Furthermore, Eq. (10) makes clear that the inverse of the parameter $\mu$ plays the role of a cutoff time, limiting the growth of $\left(\partial_{\lambda} H\right)(t)$ in the operator space. Note that this time is much longer than the timescales generally studied in the literature (e.g., the timescale characterizing the ballistic propagation of information $t_{L R}=L / v_{L R}$, where $v_{L R}$ is the Lieb-Robinson velocity and $L$ is the system size) [52-54]. One of the outcomes of our work is that an exponential sensitivity to detecting the onset of chaos requires access to exponentially long timescales (Fig. 1).

\section{NUMERICAL RESULTS}

We can now compare with results for the AGP in integrable or nonergodic models. Specifically, we move to the analysis of the norm of the regularized AGP for a specific integrable $X X Z$ model with open boundary conditions [71-75], whose Hamiltonian is given below:

$$
H_{X X Z}=\sum_{i=1}^{L-1}\left(\sigma_{i+1}^{x} \sigma_{i}^{x}+\sigma_{i+1}^{y} \sigma_{i}^{y}\right)+\Delta \sum_{i=1}^{L-1} \sigma_{i+1}^{z} \sigma_{i}^{z} .
$$

We now consider the effects of various integrabilitybreaking terms. Although the thermodynamics of the above model can be solved exactly using the Bethe ansatz [71-75], we still do not have access to matrix elements of general local operators $\left\langle n\left|\partial_{\lambda} H\right| m\right\rangle$, and the exact AGP remains out of reach even in the integrable limit. Consequently, there are also no results on the scaling of the AGP with increasing system size.

For reference, we also analyze an Ising model in the presence of a longitudinal field whose Hamiltonian is given below:

$$
H_{\text {Ising }}=\sum_{i=1}^{L-1} \sigma_{i+1}^{z} \sigma_{i}^{z}+h_{z} \sum_{i=1}^{L} \sigma_{i}^{z}+h_{x} \sum_{i=1}^{L} \sigma_{i}^{x},
$$

where open boundary conditions are chosen for the chaotic Ising model. This model has a trivially integrable limit at zero longitudinal field $h_{z}=0$, which maps to a system

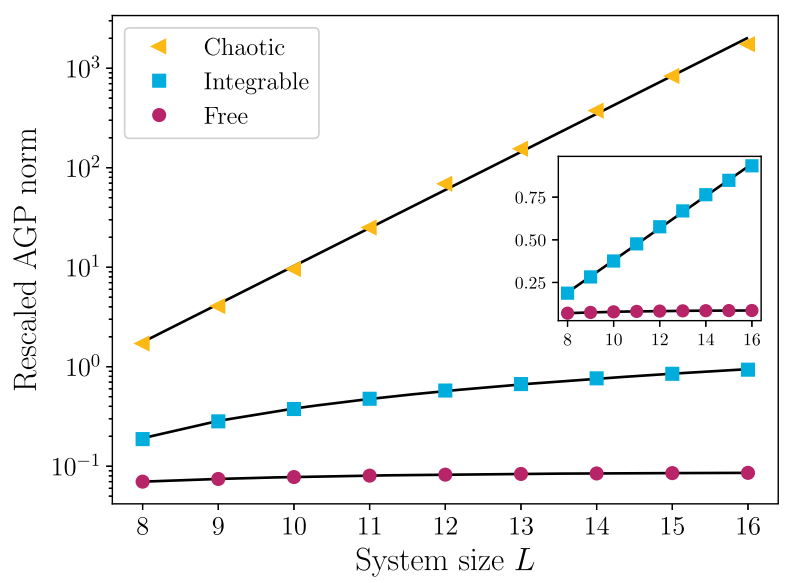

FIG. 2. AGP scaling. The rescaled norm $\left\|\mathcal{A}_{\lambda}\right\|^{2} / L$ is presented as a function of the system size for the chaotic Ising model (yellow triangles), the integrable interacting $X X Z$ model (blue squares), and the integrable noninteracting Ising model (red dots). The data corresponding to the chaotic Ising and integrable $X X Z$ models are fitted to an exponential and a linear function, respectively (black lines). For the Ising models we set $\lambda=h_{x}$, and for the $X X Z$ model we set $\lambda=\Delta$. Inset: Rescaled AGP norm for the free and interacting integrable models on a linear graph. Parameters: $h_{x}=0.8$ for the free model, $\Delta=1.1$ for the integrable model, and $h_{x}=(\sqrt{5}+5) / 8$ and $h_{z}=(\sqrt{5}+1) / 4$ for the chaotic model. $\left\|\mathcal{A}_{\text {chaotic }}\right\|^{2} \sim e^{0.9 L}$ and $\left\|\mathcal{A}_{\text {int }}\right\|^{2}=$ $0.09 L-0.56$.

of free fermions [76]. In this noninteracting (free) limit, the AGP can be computed analytically $[39,77]$ (see Appendix B). In the presence of the longitudinal field, this model shows a Wigner-Dyson-type distribution of the energy-level spacings, which is particularly pronounced at the parameters $h_{x}=(\sqrt{5}+5) / 8$ and $h_{z}=(\sqrt{5}+1) / 4$ [78]. We use these values when computing the AGP in the chaotic regime.

In Fig. 2, we show the AGP norm scaled by the system size $\left\|\mathcal{A}_{\lambda}\right\|^{2} / L$ [79] for the interacting $X X Z$ model and the Ising model at both the chaotic and noninteracting points. Figure 2 clearly shows the remarkably different scalings with system size $L$ for chaotic, integrable, and free models. For chaotic models, the scaled AGP norm shows the exponential scaling expected from the ETH. For the free model, the scaled norm is system-size independent up to exponentially small corrections away from the critical point (see Appendix B). For the integrable $X X Z$ model, the scaled AGP norm shows a nontrivial polynomial scaling: $\left\|\mathcal{A}_{\lambda}\right\|^{2} / L \propto L^{\beta}$. We find that the exponent $\beta$ is nonuniversal and depends on the choice of the anisotropy $\Delta$ (see Appendix D). We choose $\lambda=h_{x}$ for both the integrable and nonintegrable Ising models and $\lambda=\Delta$ for the $X X Z$ model.

While the exponential scaling of the AGP norm in the chaotic regime and the constant AGP norm in the free model are expected, the polynomial scaling of this norm of the $X X Z$ integrable model is very interesting and leads to 
nontrivial conclusions. Recently, LeBlond et al. [61] have shown that the matrix elements of local operators in this integrable model are not sparse (as compared to the matrix elements of noninteracting integrable models). The latter implies that Eq. (6) for the AGP norm still applies, where $\left|f_{\lambda}(\omega)\right|^{2}$ can also be found from the Fourier transform of the symmetric correlation function (see Appendix C). Since we choose $\mu$ to be exponentially small in the system size and $\left\|\mathcal{A}_{\lambda}\right\|^{2}$ is polynomially (not exponentially) large, the function $f_{\lambda}(\omega)$ must vanish as $\omega \rightarrow 0$. This behavior is to be contrasted with chaotic systems, where at small $\omega$ this function saturates at a constant value, in agreement with the random matrix theory [28].

\section{INTEGRABILITY BREAKING}

Having established the scaling of the AGP norm in three different regimes, we move to the analysis of integrability breaking by small perturbations and focus on a more generic $X X Z$ model. As an integrability-breaking term, we choose a magnetic field coupled to a single spin in the middle of the chain, acting as a single-site defect:

$$
V=\sigma_{\lceil(L+1) / 2\rceil}^{z},
$$

where $\lceil(L+1) / 2\rceil$ stands for the smallest integer greater than or equal to $(L+1) / 2$. Then, we analyze the AGP for the total Hamiltonian

$$
H=H_{X X Z}+\epsilon_{d} V
$$

as a function of the integrability-breaking parameter $\epsilon_{d}$. Interestingly, in Ref. [80], it is argued based on the same model that even a single-site defect is sufficient to induce chaos in the thermodynamic limit. In Appendix E, we analyze an extensive integrability-breaking perturbation by considering $H=H_{X X Z}+\Delta_{2} V$ with $V=\sum_{i} \sigma_{i+2}^{z} \sigma_{i}^{z}$ and find the results to be consistent. The similarity between the effects of local and global perturbations on spectral properties is also found in Ref. [81].

A challenging question is how quickly chaos emerges when a nonergodic or integrable system is subjected to an integrability-breaking perturbation. In classical systems with few degrees of freedom, it is known from Kolmogorov-Arnold-Moser theory that integrable systems are stable against small perturbations [82-84]. It is widely believed that quantum chaos is generally induced by infinitesimal perturbations in the thermodynamic limit $[47,48,85,86]$, with the potential exception of many-body localization $[87,88]$, although the precise scaling of the critical perturbation strength with the system size remains an open question. A standard limitation of numerical approaches (using, e.g., level statistics or spectral form factor) addressing this question is the small system sizes amenable to simulations, where it is possible to reliably extract the data.

In Fig. 3(a), we show the scaling of the norm of the AGP as a function of the system size for different perturbation strengths $\epsilon_{d}$. We choose the zero magnetization subspace of the $X X Z$ chain with the number of spins up $N_{\uparrow}=\lfloor L / 2\rfloor$, where $\lfloor L / 2\rfloor$ stands for the largest integer less than or equal to $L / 2$, and for the direction of the AGP we choose $\lambda=\Delta$, i.e., as in Fig. 2. For the cutoff, we choose $\mu=L \mathcal{D}_{0}^{-1}$, where $\mathcal{D}_{0}$ is the dimension of zero magnetization sector. From the figure, we clearly see a sharp crossover in the scaling of the norm of the AGP as a function of the system size from the integrable power law behavior to the chaotic exponential behavior. The straight lines are obtained by a least squares fit, with the slope extracted for the largest $\epsilon_{d}$ and then used for other perturbations. After the best fitting parameters are found, the critical system sizes are obtained for a particular defect energy at which the integrable (polynomial) and chaotic (exponential) curves intersect. These values are shown in the inset in Fig. 3(a), showing a clear exponential scaling of the critical perturbation strength with the system size. Interestingly, the slope of the exponential scaling $\beta \approx 1.28$ is almost twice the slope predicted by the ETH, $\beta=\log (2) \approx 0.69$. Notably, the slope of $2 \log (2)$ is the largest possible growth rate of the AGP norm (see Appendix C). In the next section, we return to this point and relate it to the emergence of relaxation times that are exponentially long in system size.

Consistent results are obtained for the Ising model (13), where one can consider breaking the integrability of the transverse field Ising model $\left(h_{z}=0\right)$ by introducing a small nonzero $h_{z}$ field, while probing the integrable direction $\lambda=h_{x}$. The results are shown in Fig. 3(b). As in the $X X Z$ case, we observe a sharp crossover from the unperturbed scaling of the AGP norm (see Fig. 2) to exponential scaling with an exponent that exceeds the ETH expectation, once again having implications on the long time relaxation of the system.

To contrast the scaling of the AGP norm with more traditional approaches in Fig. 4, we show the mean ratio of energy-level statistics as a function of the defect energy for system size $L=16$. Given subsequent energy-level spacings $s_{n}=E_{n+1}-E_{n}$, this ratio is defined as

$$
r_{n}=\frac{\min \left(s_{n}, s_{n+1}\right)}{\max \left(s_{n}, s_{n+1}\right)} .
$$

For nonergodic systems and Poissonian level statistics $\langle r\rangle \approx 0.386$, whereas for chaotic systems and WignerDyson statistics $\langle r\rangle \approx 0.536$. In this model, the average ratio $\langle r\rangle$ shows the crossover from nonergodic to ergodic behavior at $\epsilon_{d}^{*} \sim 0.1$ [89]. This crossover value of $\epsilon_{d}$ has a very weak dependence on the system size. In comparison, for the same system size $L=16$, the AGP norm shows a clear crossover to chaos for a much smaller $\epsilon_{d}^{*} \sim 10^{-3}$ [see Fig. 3(a)]. For larger system sizes, the gap between the 


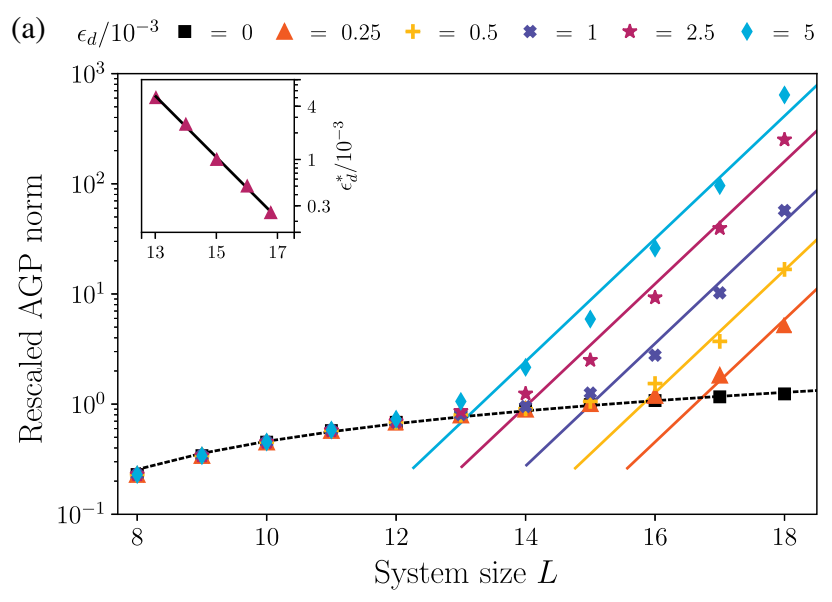

(b)

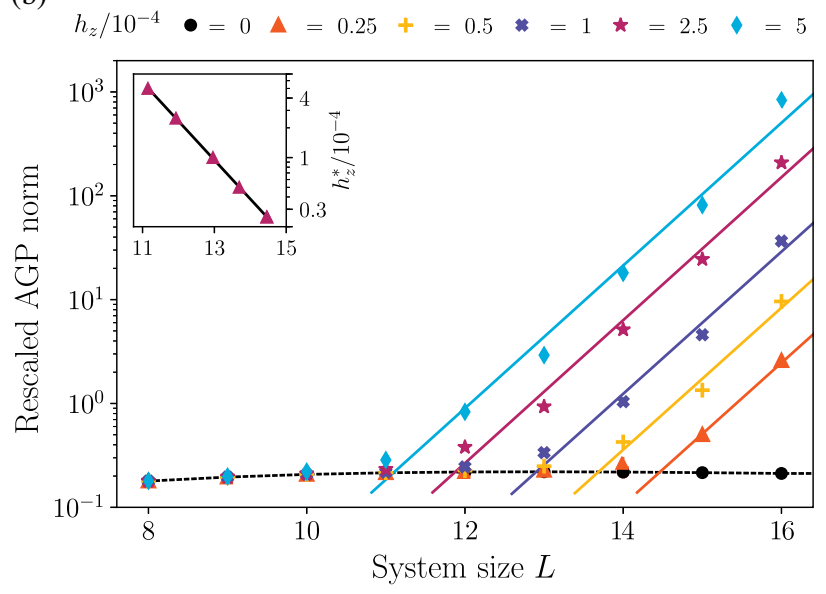

FIG. 3. Integrability breaking. The rescaled AGP norm $\left\|\mathcal{A}_{\lambda}\right\|^{2} / L$ of (a) the $X X Z$ chain with $\lambda=\Delta$ and (b) the Ising chain with $\lambda=h_{x}$. Both models show a sharp crossover from polynomial to exponential scaling with system size, even for very small integrability-breaking perturbation strengths. With decreasing perturbation strength, the system size where this crossover happens increases. Straight lines are the exponential fits with $\left\|A_{\lambda}\right\|^{2} / L \sim e^{\beta L}$, where $\beta=1.28$ for the $X X Z$ and $\beta=1.58$ for the Ising model. The insets show the scaling of the crossover point, i.e., the dependence of the integrability-breaking perturbation on system size. The critical perturbation strength scales exponentially with system size, with $\epsilon_{d}^{*} \sim e^{-0.8 L}$ for the $X X Z$ chain and $h_{z}^{*} \sim e^{-0.9 L}$ for the Ising chain. Parameters: (a) $\Delta=1.1$ and (b) $h_{x}=0.75$.

chaos thresholds extracted by these two methods becomes even larger. Moreover, we also estimate the critical perturbation strength using the spectral form factor for the same system size $L=16$. Since this estimation generally does not self-average $[90,91]$, we add disorder to the $z z$ coupling in the Hamiltonian [Eq. (15)], which reduces the sensitivity of this probe to detect chaos. From the spectral form factor, we find $\epsilon_{d}^{*} \sim 0.1$, a value where the level statistics is roughly halfway between Poisson and WignerDyson (see Fig. 4). Such a correspondence is also observed for disordered models in Ref. [46].

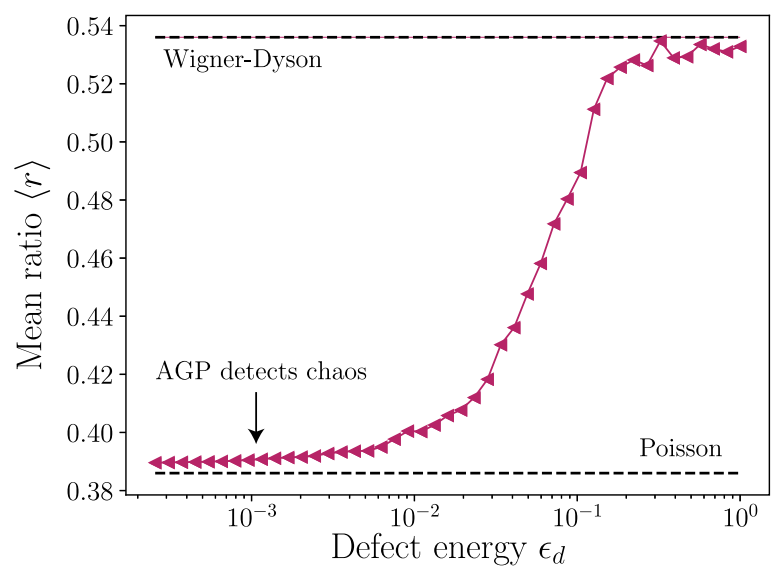

FIG. 4. Energy-level statistics. Mean ratio of energy-level spacings $\langle r\rangle$ as a function of defect energy $\epsilon_{d}$ for an $X X Z$ chain of length $L=16$ at anisotropy $\Delta=1.1$. The arrow indicates the value of the defect energy where chaos can be detected (for $L=16$ ) using the exponential scaling of the AGP norm.

We believe that the reason that the AGP norm is so much more sensitive is that it effectively detects the change in the differential of the norm with the system size. The absolute value of the AGP norm at the threshold is still much closer to the integrable value than to the chaotic one. Such a differential is much harder to detect using other measures, e.g., the level spacing ratio, because this crossover is much smoother, and it is harder to define a sharp threshold.

In Fig. 5, we show similar results, now choosing to deform the Hamiltonian in the direction of the integrabilitybreaking operator itself, i.e., $\lambda=\epsilon_{d}$ for the $X X Z$ chain and $\lambda=h_{z}$ for the Ising chain. We choose to work in the full Hilbert space with dimension $\mathcal{D}=2^{L}$. We find that the AGP norm shows exponential scaling even when $\epsilon_{d}=0$,

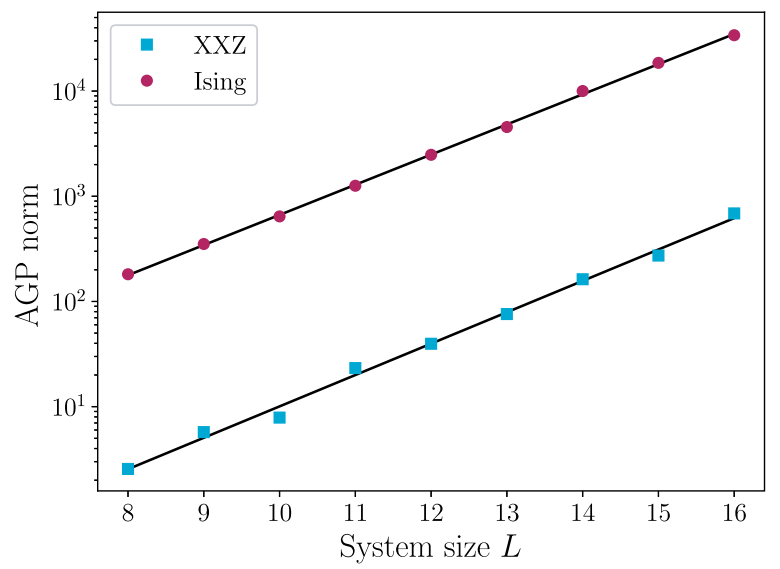

FIG. 5. Integrability-breaking deformation at the integrable point. The AGP norm $\left\|\mathcal{A}_{\lambda}\right\|^{2}$ shows an exponential scaling at the integrable point for the $X X Z$ chain (squares) with $\lambda=\epsilon_{d}$ and the Ising chain (circles) with $\lambda=h_{z}$. The black lines correspond to exponential fits, i.e., $\left\|\mathcal{A}_{\lambda}\right\|^{2} \sim e^{\beta L}$, where $\beta \approx \log (2)$. $X X Z$ parameters: $\Delta=1.1$ and $\epsilon_{d}=0$. Ising parameters: $h_{x}=0.75$ and $h_{z}=0$. 
i.e., when the Hamiltonian is integrable. We find a good fit to the exponential scaling $\left\|\mathcal{A}_{\lambda}\right\|^{2} \sim e^{\beta L}$, with now $\beta \approx \log (2)$. Again, we confirm that the results remain the same if we use an extensive integrability-breaking term instead (see Appendix F).

\section{LONG RELAXATION TIMES}

We already mentioned a very peculiar fact following from Fig. 3: Namely, instead of the perhaps expected crossover of the integrable polynomial scaling of the AGP norm to the ETH exponential scaling with the slope $\log (2)$, the AGP crosses over to the exponential scaling regime with the slope $\beta=1.28$, which is almost twice as large as the slope predicted by the ETH, $\beta=\log (2) \approx 0.69$. Combining this result with Eq. (6), which we highlight works in both integrable and nonintegrable regimes, we conclude that at small $\omega$ the function $\left|f_{\lambda}(\omega)\right|^{2}$ should scale exponentially with the system size. This conclusion implies that the system must have exponentially long relaxation times, which are known to exist in classical chaotic systems like the FPUT chain [49-51]. Although we cannot rule out the eventual relaxation to the ETH value for system sizes greater than those we study, our results here suggest that, while an exponentially small perturbation is sufficient to induce chaos in the system, it takes an exponentially long time for the system to relax to the steady state. In Appendix E, we show that a similar behavior persists if we break the integrability by a small extensive perturbation, here chosen as the second-nearest-neighbor Ising interactions. We find the same slope of $\beta \approx 1.28$, ruling out that this scaling is induced by the ultralocal nature of the perturbation in Fig. 3(a). As the defect energy is increased further to large values (in particular, $\epsilon_{d} \sim 1$ ), we find that the slope of the AGP norm's exponential growth reduces again to the ETH value of $\beta \approx \log (2)$ (see Appendix F).

To make the connection between the AGP norm and the relaxation time more explicit, let us observe that from Eq. (6) for sufficiently small $\mu$ one can make the following estimate:

$$
\|\left.\mathcal{A}_{\lambda}\right|^{2} \sim \frac{\left|f_{\lambda}(\mu)\right|^{2}}{\mu} .
$$

For integrable directions $\lambda$ (e.g., $\lambda=\Delta$ for the $X X Z$ model) and $L>L^{*}$, where the AGP norm has exponential scaling, the norm becomes

$$
\|\left.\mathcal{A}_{\lambda}\right|^{2} \sim C e^{\beta\left(L-L^{*}\right)}
$$

where $C$ roughly is the value of the unperturbed AGP norm at $L^{*}$. Recall that we observe a scaling of the critical perturbation strength like $\epsilon_{d} \sim e^{-\alpha L^{*}}$, such that one finds

$$
\left|f_{\lambda}(\mu)\right|^{2} \sim C \mu e^{\beta\left(L-L^{*}\right)} \sim C \epsilon_{d}^{\eta} e^{\kappa L},
$$

where $\eta=\beta / \alpha, \kappa=\beta-\log (2)$, and we neglect all polynomial factors in system size. For the $X X Z$ model, the exponents are $\eta \approx 1.6$ and $\kappa \approx 0.85 \log (2)$ (see the caption of Fig. 3). Because $\left|f_{\lambda}(\omega)\right|^{2}$ is the Fourier transform of the two-point correlation function of $\partial_{\lambda} H$ [see Eq. (8)], as $\omega \rightarrow 0$, it is proportional to the relaxation time of the system. Combining these considerations, we see that for the $X X Z$ model we have

$$
\tau \sim \epsilon_{d}^{\eta} e^{\kappa L},
$$

with both $\kappa$ and $\eta$ of $O(1)$. Similarly, for the Ising model, $\tau \sim h_{z}^{\eta} e^{\kappa L}$, where $\eta \approx 1.8$ and $\kappa \approx 1.28 \log (2)$ (see the caption of Fig. 3). We see that the relaxation time increases exponentially with the system size. For large system sizes, it can saturate at some $L$-independent value, which should diverge as $\epsilon_{d} \rightarrow 0$. This result would reflect the crossover of the scaling of the AGP norm to the ETH result: $\left\|A_{\lambda}\right\|^{2} \propto \exp [S(L)]=\exp [\log (2) L]$. While this scenario seems likely, we do not see any signatures for such a crossover within our numerics and, thus, cannot rule out more exotic scenarios for the behavior of the relaxation time with the system size. Moreover, at intermediate system sizes accessible to our numerics, we see an extremely stable exponential scaling of the AGP norm (and, hence, of the relaxation time), with the exponent $\beta$ independent of the strength of the integrability-breaking perturbation as long as it is sufficiently small. Interestingly, in a follow-up work [92], a similar exponential scaling of the AGP norm with $\beta \approx 2 \log (2)$ is observed in a disordered central spin model even in the absence of any small parameters, i.e., at large integrability-breaking perturbations. We note that, in all the systems analyzed so far in this regime, $\beta$ saturates near the maximum allowed value $2 \log (2)$, within numerical

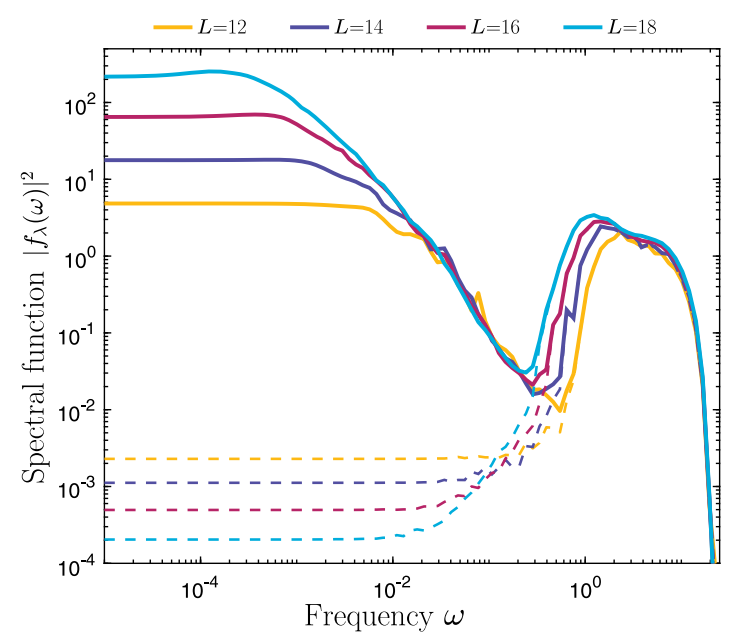

FIG. 6. The spectral weight for the integrable perturbation. The spectral weight $\left|f_{\lambda}(\omega)\right|^{2}$ for the integrable perturbation $\lambda=\Delta$ in the $X X Z$ model at small integrability-breaking perturbation $\epsilon_{d}=$ 0.05 (solid lines) and at the integrable point $\epsilon_{d}=0$ (dashed lines). The remaining parameters are the same as in Fig. 3. 
precision. From the point of view of operator spreading, this value is very reminiscent of the $2 \log (2)$ scaling of the operator entanglement entropy in maximally chaotic dualunitary models [93]. Whether it is a simple coincidence or there is a deeper connection remains to be understood.

To illustrate these general considerations about the relaxation times, we extract the function $\left|f_{\lambda}(\omega)\right|^{2}$ directly. Usually, it is very difficult to do so at exponentially small frequencies of interest, since there are very few eigenstates involved, hence leading to large fluctuations. Here, we compute $\left|f_{\lambda}(\omega)\right|^{2}$ by replacing all the delta functions in Eq. (8) with Lorentzians of width $\mu$. In all the figures, $\mu=L 2^{-L}$, consistent with the AGP regularization. The total spectral weight is subsequently computed on a
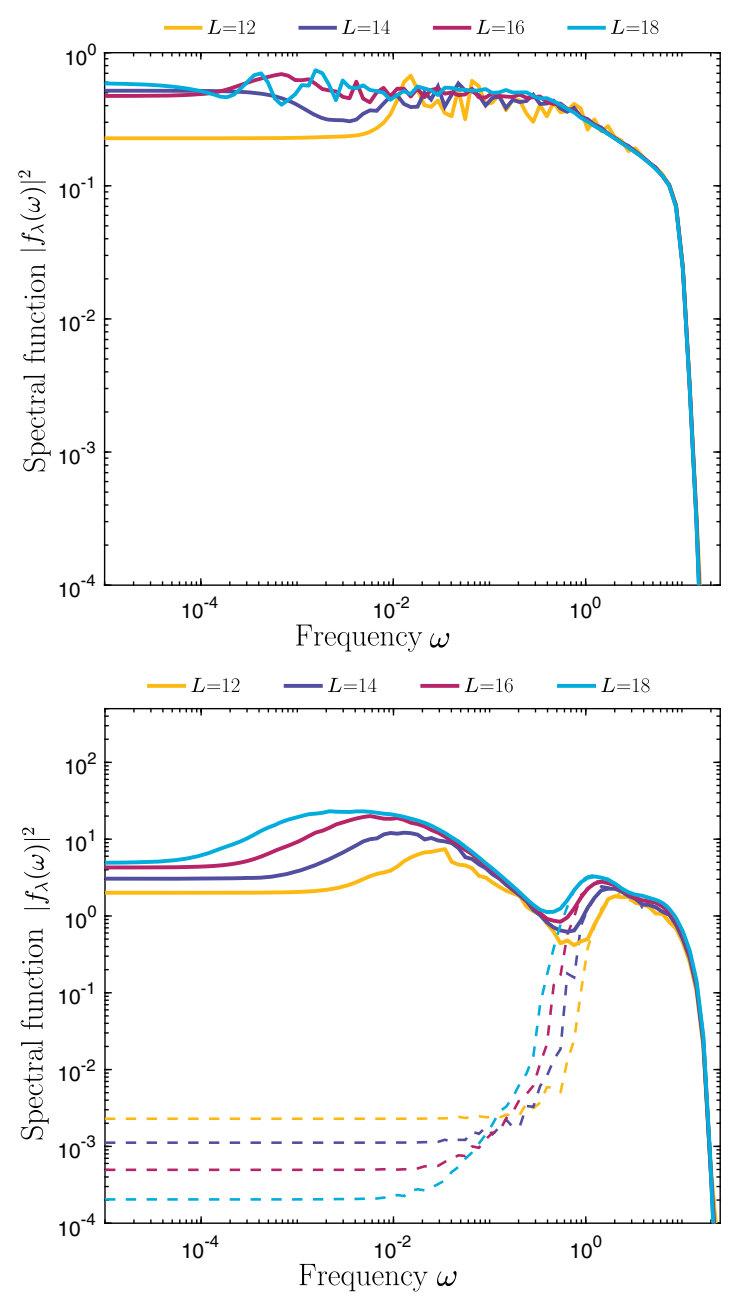

FIG. 7. The spectral weights for the nonintegrable perturbation. The spectral weight $\left|f_{\lambda}(\omega)\right|^{2}$ for the nonintegrable perturbation $\lambda=\epsilon_{d}$ in the $X X Z$ model at the integrable point $\epsilon_{d}=0$ (top) and for the perturbation $\lambda=\Delta$ at the strongly nonintegrable point, i.e., in the ETH regime, $\epsilon_{d}=0.5$ (bottom). The dashed lines in the bottom are the result at the integrable point $\epsilon_{d}=0$, showing the high-frequency behavior remains unmodified even at these large perturbation strengths. The remaining parameters are the same as in Fig. 6. logarithmically spaced grid. All the figures show the average spectral weight in each bin.

In Fig. 6, we show the extracted spectral weight $\left|f_{\lambda}(\omega)\right|^{2}$ for the $X X Z$ model with $\lambda=\Delta$ for a small integrabilitybreaking perturbation $\epsilon_{d}=0.05$ (solid lines) and exactly at the integrable point $\epsilon_{d}=0$ for four different system sizes $L=12,14,16,18$. As predicted from the AGP scaling, there is a clear exponentially growing spectral weight at small frequencies with an exponentially shrinking frequency range, where it plateaus. In the integrable regime, conversely, $\left|f_{\lambda}(\omega)\right|^{2}$ is exponentially decreasing with the system size, approaching zero in the thermodynamic limit.

To contrast this behavior of the spectral function with the other two regimes where the AGP norm shows exponential scaling with $\beta=\log (2)$, in Fig. 7 , we show $\left|f_{\lambda}(\omega)\right|^{2}$ in such regimes. The top shows the $\left|f_{\lambda}(\omega)\right|^{2}$ for the nonintegrable perturbation $\lambda=\epsilon_{d}$ at the integrable point of the $X X Z$ model $\epsilon_{d}=0$, while the bottom corresponds to the perturbation $\lambda=\Delta$ at the strongly nonintegrable point $\epsilon_{d}=0.5$ where the system satisfies the ETH $[32,63]$.

\section{DISTINGUISHING BETWEEN INTEGRABLE AND ETH REGIMES}

The AGP clearly depends on both the Hamiltonian $H$ and the direction along which it is deformed, i.e., $\partial_{\lambda} H$. In the previous sections, we argue that generic perturbations in chaotic systems lead to an AGP norm scaling exponentially with the system size, whereas in integrable models integrability-preserving perturbations lead to an AGP norm scaling polynomially. This scaling is directly reflected in the relaxation times of $\partial_{\lambda} H$ through its probing of the zerofrequency limit of $\left|f_{\lambda}(\omega)\right|^{2}$. However, in specific cases, polynomial scaling of the gauge potential can also be observed in chaotic systems.

In particular, there is a special class of operators which can be represented as $K=i[H, B]$, where $B$ is a local operator or a sum of local operators. A current can, e.g., be represented in this way as $B=\sum_{i} i n_{i}$, where $n_{i}$ is the conserved charge; $n_{i}=\sigma_{z}^{i}$ for the $X X Z$ model. For such operators, $\mathcal{A}_{\lambda}=B$ by construction, and the AGP has a polynomial norm irrespective of whether the system is integrable or chaotic. For such operators, $\left|f_{\lambda}(\omega)\right|^{2}$ must also vanish at $\omega \rightarrow 0$, consistent with recent numerical results [63]. On a related note, see Ref. [94]. Physically, this nondivergence of the AGP, even in the chaotic systems satisfying the ETH, simply follows from the fact that deforming the Hamiltonian with the operator $K$ is a symmetry transformation, which does not change the spectrum of the Hamiltonian but simply transforms the eigenstates with the unitary operator $U=\exp (-i \lambda B)$. When checking for quantum chaos, such deformations can be explicitly excluded when probing the scaling of the gauge potential. 
While the existence of nontrivial deformations with polynomial scaling of the AGP norm is an indicator of integrability, generic integrability-breaking perturbations give rise to exponential scaling, in which case the specific dependence on $\mu$ offers further information. Note that this indicator also implies the existence of a family of integrable models, excluding more exotic "isolated" integrable systems where every possible perturbation breaks integrability.

In the previous section, the scaling of the AGP norm is the same as one would expect from the ETH, even though at $\epsilon_{d}=0$ the system is integrable and the ETH is clearly violated. The non-ETH behavior can be seen, e.g., in large eigenstate-to-eigenstate fluctuations of the expectation value of $\sigma_{\lceil(L+1) / 2\rceil}^{z}$ [64]. For this perturbation, the scaling of the AGP with the system size simply tells us that $\left|f_{\lambda}(\omega)\right|^{2}$, which remains well defined in such models, saturates to a nonzero constant at small $\omega$, as confirmed directly in the previous section. Similar to the usual matrix elements of observables, the information about the integrability of the system is now contained in the statistical properties of the AGP norm.

More specifically, for random matrix ensembles, the statistical properties of the fidelity susceptibility (equivalent to the contributions to the AGP norm for individual eigenstates) are analyzed in Ref. [95], where the distribution for different eigenstates is considered. The fidelity susceptibility $z_{n, \lambda}$ of an eigenstate $|n(\lambda)\rangle$ is equivalent to

$$
z_{n, \lambda} \equiv \frac{1}{\mathcal{D}}\left\langle n\left|\mathcal{A}_{\lambda}^{2}\right| n\right\rangle_{c} \equiv \frac{1}{\mathcal{D}} \sum_{m \neq n}\left|\left\langle n\left|\mathcal{A}_{\lambda}\right| m\right\rangle\right|^{2},
$$

such that $\left\|\mathcal{A}_{\lambda}\right\|^{2}=\sum_{n} z_{n, \lambda}$.

Let us briefly present a simple derivation of the tail of this distribution and then contrast the AGP distribution for integrable and ETH regimes. The tail of this distribution for typical (random) perturbations is dominated by contributions from neighboring energy levels, such that its distribution inherits its properties from the level spacing distribution.

Recall that the exact AGP norm with $\mu=0$ is given by Eqs. (2) and (3). For a typical perturbation, we can replace the numerator of Eq. (2) with a random matrix such that typical matrix elements are of the order of $1 / \sqrt{\mathcal{D}}$ [see Eq. (8)]. The tail of the distribution for large $z_{n, \lambda}$ is dominated by nearby energy levels, and we can approximate

$$
z_{n, \lambda} \approx \frac{C}{s_{n}^{2}},
$$

where $s_{n}$ is the level spacing $E_{n+1}-E_{n}$ now normalized by the Hilbert space dimension (such that the mean value of $s$ is unity) and $C$ is an unimportant constant, which we can set to one. The scaling of the probability distribution at large $z_{\lambda}$ follows as

$$
\operatorname{Pr}\left(z_{\lambda}=x\right) \sim \frac{1}{x^{3 / 2}} P\left(\frac{1}{\sqrt{x}}\right),
$$

where $P(s)$ is the normalized nearest-neighbor level spacing distribution.

For integrable systems, there is no level repulsion, $P(s \rightarrow 0) \neq 0$, and we have (to dominant order)

$$
\operatorname{Pr}\left(z_{\lambda}=x\right) \propto x^{-3 / 2}
$$

for $x \gg 1$. Note that, as a consequence of this fat tail, the mean AGP diverges without regularization. The regularization with $\mu$ in the norm of the AGP effectively introduces a cutoff to the energy denominator at the rescaled cutoff $\bar{\mu}=\mu \mathcal{D}$. Assuming that the AGP norm is dominated by the contributions $z_{n, \lambda}$ for which the derived scaling holds, we can say that the average fidelity susceptibility is given by $\left\langle z_{\lambda}\right\rangle \propto 1 / \bar{\mu}$, and, hence, $\left\|\mathcal{A}_{\lambda}\right\|^{2}=\mathcal{D}\left\langle z_{\lambda}\right\rangle \sim \mathcal{D} / \bar{\mu}$. This result agrees with the observed scaling shown in Fig. 5. On the other hand, chaotic systems satisfying the ETH exhibit level repulsion and $P(s) \approx s^{\beta}$, resulting in $\operatorname{Pr}\left(z_{\lambda}=x\right) \propto$ $x^{-(3+\beta) / 2}$ at large values of $x$. For the considered Ising and $X X Z$ model, the relevant random matrix ensemble is a Gaussian orthogonal ensemble, for which $\beta=1$ and

$$
\operatorname{Pr}\left(z_{\lambda}=x\right) \propto x^{-2} .
$$

In contrast to the integrable model, the mean $\left\langle z_{\lambda}\right\rangle \sim$ $-\log \left(\bar{\mu}^{2}\right)$ diverges only logarithmically with the cutoff. These simple scaling arguments agree very well with numerical observations shown in Fig. 8.

From this analysis, we can conclude that choosing a fixed $\mu \sim 1 / \mathcal{D}$ leads to the same scaling of the AGP norm

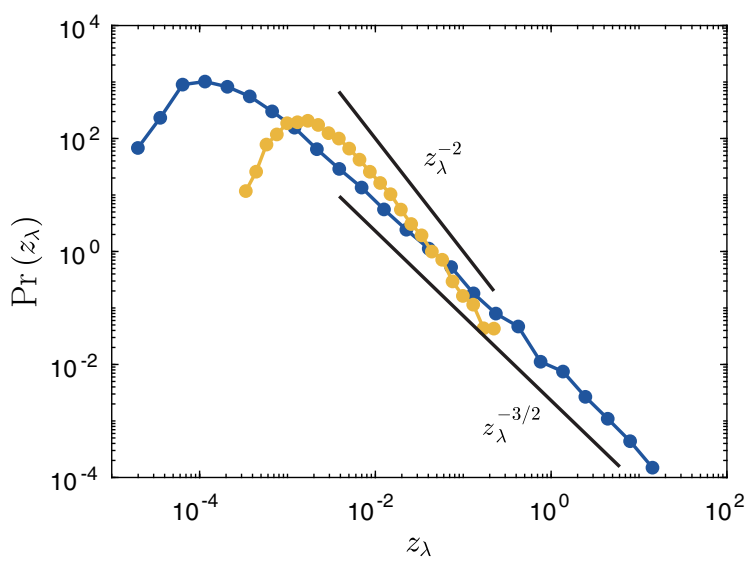

FIG. 8. AGP norm distribution. Distribution of the eigenstate contributions $z_{\lambda}$ to the rescaled AGP norm [see Eq. (22)] for the $X X Z$ model with $L=16$ spins. The two curves describe the results for the nonintegrable perturbation $\lambda=\epsilon_{d}$ at the integrable point $\epsilon_{d}=0$ (blue) and for the perturbation $\lambda=\Delta$ at the strongly nonintegrable point $\epsilon_{d}=0.5$ (yellow). Black lines show the expected scalings $z_{\lambda}^{-3 / 2}$ and $z_{\lambda}^{-2}$ for the integrable and nonintegrable model, respectively. 
with the Hilbert space dimension for the integrable model with a chaotic deformation $\lambda$ and for the ergodic ETH model. However, these two limits can still be distinguished by either the different scaling of the AGP norm with the cutoff $\mu$ or, equivalently, by the presence of an exponentialin-system-size difference between the typical and the average contributions of individual states to the AGP norm in the former (integrable) regime and the lack of such an exponential difference in the latter $(\mathrm{ETH})$ regime.

\section{CONCLUSIONS}

We found that the properly regularized norm of the adiabatic gauge potential, the generator of adiabatic deformations, can serve as an extremely sensitive probe of quantum chaotic behavior. Within chaotic systems, this norm scales exponentially with the system size, whereas it scales polynomially in interacting integrable systems and is approximately system-size independent in noninteracting systems for adiabatic deformations preserving integrability. For adiabatic deformations breaking integrability, exponential scaling is generally observed.

Using the present method to investigate the effects of an integrability-breaking perturbation on the $X X Z$ and Ising chains, we found that perturbations that are exponentially small in system size suffice to induce chaotic behavior. We also found that such a small integrability-breaking term leads to anomalously slow dynamics along the integrable directions, with the relaxation time scaling exponentially with the system size. Such integrability-breaking perturbations can also be detected at the integrable point, where no anomalous dynamics occur. Even though typical perturbations show exponential scaling of the regularized norm of the adiabatic gauge potential, regardless of whether the system is integrable or not, one can distinguish the two cases by their dependence on the regularization parameter or by their fluctuations.
This result motivates the use of the adiabatic gauge potential, which is connected with both deformations of eigenstates and operator dynamics, as a sensitive probe into either chaotic or integrable behavior of quantum manybody systems.

\section{ACKNOWLEDGMENTS}

Some of the numerical computations were performed using Quspin [96,97]. We thank Marcos Rigol and Lea Santos for detailed and very useful feedback on the manuscript. We also thank Anushya Chandran, Anatoly Dymarsky, Phil Crowley, Pranay Patil, Lev Vidmar, Tamiro Villazon, Phil Weinberg, and Jonathan Wurtz for useful discussions. We also acknowledge technical support by Boston University's Research Computing Services. M. P. and D. K. C. acknowledge support from Banco Santander Boston University-National University of Singapore grant. P. W. C. gratefully acknowledges support from a Francqui Foundation fellowship from the Belgian American Educational Foundation (BAEF), Boston University's Condensed Matter Theory Visitors program, and EPSRC Grant No. EP/P034616/1. A. P. was supported by the NSF Grant No. DMR-1813499 and the AFOSR Grant No. FA9550-16-1-0334. D. S. acknowledges support from the FWO as postdoctoral fellow of the Research Foundation-Flanders.

\section{APPENDIX A: CUTOFF SCALING WITH SYSTEM SIZE}

Unless stated otherwise, in all calculations we choose a cutoff $\mu=L \mathcal{D}^{-1}$, where $\mathcal{D}$ is the dimension of the Hilbert space. The prefactor $L$ is chosen to remove the logarithmic correction coming from the zero-frequency contribution of $|f(\omega=0)|^{2}=L$ in chaotic models (see Appendix C). This choice can also be motivated by plotting the AGP norm and comparing it with respect to different choices of cutoff. (a)

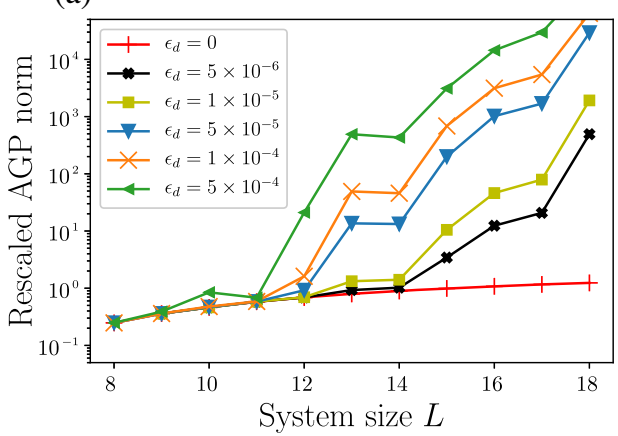

(b)

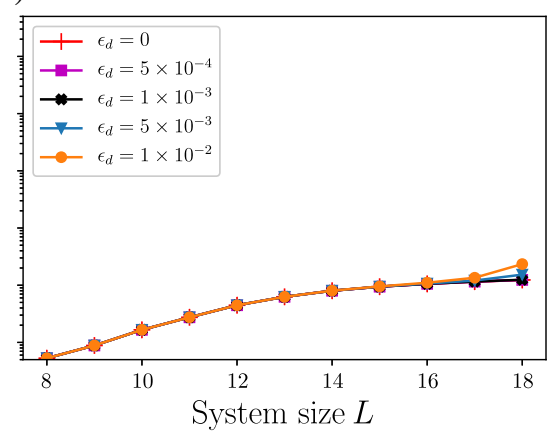

(c)

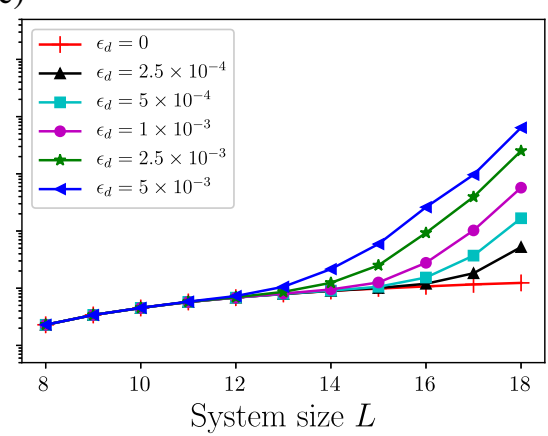

FIG. 9. Effects of regularization. Size dependence of the rescaled AGP norm $\left\|A_{\lambda}\right\|^{2} / L$ for different choices of scaling for the cutoff $\mu$ close to chaotic-integrable transition point: (a) When $\mu=L^{-1 / 2} \mathcal{D}_{0}^{-1}$, where $\mathcal{D}_{0}$ is the dimension of zero magnetization sector, the variation of the norm with the system size is noisy. (b) When $\mu=L^{2} \mathcal{D}_{0}^{-1}$, the norm, albeit smooth, is no longer very sensitive to small integrability-breaking perturbations. (c) When $\mu=L \mathcal{D}_{0}^{-1}$, the norm is both appropriately smooth and exponentially sensitive to integrability-breaking perturbations. Model: $X X Z$ chain with a defect in the middle [Eq. (15)]. Parameters: $\Delta=1.1$ and $\lambda=\Delta$. 
We first study this norm close to the chaotic-integrable transition point and then later describe its effect deep in the chaotic regime.

When we are close to the chaotic-integrable transition point and the cutoff is too small (e.g., $\mu=L^{-1 / 2} \mathcal{D}^{-1}$ ), then we find that the AGP norm is too sensitive to the exponentially close eigenstates, showing a nonsmooth exponential scaling, which makes it hard to draw any conclusions [see Fig. 9(a)]. On the other hand, if the cutoff is too large (e.g., $\mu=L^{2} \mathcal{D}^{-1}$ ), then the AGP norm, albeit smooth, is no longer sensitive to the small strength of integrability-breaking perturbations [see Fig. 9(b)]. In Fig. 9(c), with $\mu=L \mathcal{D}^{-1}$, we find that the rescaled AGP norm shows an exponential scaling that is both appropriately smooth and exponentially sensitive to integrability-breaking perturbations.

Deep in the chaotic (ergodic) phase, we find that the numerically obtained scaling for the norm of the AGP is almost the same for the different choices of cutoff scaling we study.

\section{APPENDIX B: DERIVATION OF AGP FOR THE FREE MODEL}

As shown in Refs. [39,77], the AGP for changing the transverse field $h_{x}$ in a free Ising model with periodic boundary conditions is given by

$$
\mathcal{A}_{h}=\sum_{l=1}^{L} \alpha_{l} O_{l},
$$

where the operators $O_{l}$ are given by the following Pauli string operators:

$O_{l}=\sum_{j=1}^{L}\left(\sigma_{j}^{x} \sigma_{j+1}^{z} \ldots \sigma_{j+l-1}^{z} \sigma_{j+l}^{y}+\sigma_{j}^{y} \sigma_{j+1}^{z} \ldots \sigma_{j+l-1}^{z} \sigma_{j+l}^{x}\right)$

and the coefficients $\alpha_{l}$ are given by

$$
\alpha_{l}=-\frac{1}{4 L} \sum_{k=0}^{\pi(L-1) / L} \frac{\sin (k) \sin (l k)}{\left(\cos k-h_{x}\right)^{2}+\sin ^{2} k} .
$$

The norm of the AGP follows as

$$
\left\|\mathcal{A}_{h}\right\|^{2}=\frac{1}{2^{L}} \operatorname{Tr}\left[\mathcal{A}_{h}^{2}\right]=2 L \sum_{l=1}^{L} \alpha_{l}^{2},
$$

where $\operatorname{Tr}\left[O_{l} O_{p}\right]=2^{L+1} L$ is used, since all strings of Pauli matrices are trace-orthogonal. The above expression is used to compute the AGP norm for the free model in Fig. 2 in the main text.

To obtain the scaling with system size, we can use the analytical expressions of $\alpha_{l}$ for large enough system sizes [39], i.e., $\alpha_{l}=h_{x}^{-l-1}$ in the paramagnetic phase where $h_{x}^{2}>1$. Using this result, we find that

$$
\left\|\mathcal{A}_{h}\right\|^{2} \sim \frac{1}{h_{x}^{2}\left(h_{x}^{2}-1\right)} L\left(1-e^{-2 L \log h_{x}}\right) .
$$

Recall that the correlation length in the transverse field Ising model $\sim 1 / \log h_{x}$.

\section{APPENDIX C: AGP BOUND}

Recall that the norm of the AGP can be expressed as

$$
\left\|\mathcal{A}_{\lambda}\right\|^{2}=\int d \omega \frac{\omega^{2}}{\left(\omega^{2}+\mu^{2}\right)^{2}} \overline{\left|f_{\lambda}(\omega)\right|^{2}},
$$

with

$$
\overline{\left|f_{\lambda}(\omega)\right|^{2}}=\frac{1}{\mathcal{D}} \sum_{n} \sum_{m \neq n}\left|\left\langle n\left|\partial_{\lambda} H\right| m\right\rangle\right|^{2} \delta\left(\omega_{n m}-\omega\right),
$$

and $\omega_{n m}=E_{n}-E_{m}$. It follows directly from Eq. (C1), and $x^{2} /\left(x^{2}+1\right)^{2} \leq 1 / 4$, that

$$
\left\|\mathcal{A}_{\lambda}\right\|^{2} \leq \frac{1}{4 \mu^{2}} \int d \omega\left|f_{\lambda}(\omega)\right|^{2}=\frac{\left\|\partial_{\lambda} H\right\|^{2}}{4 \mu^{2}} .
$$

Consequently, for any local perturbation, the norm of the regularized AGP-where we set $\mu \sim L 2^{-L}$ — cannot grow faster than $4^{L}$. Not only does it appear that this bound is saturated when probing integrable directions $\partial_{\lambda} H$ in models in which the integrability is weakly broken, it further implies that those observables $\partial_{\lambda} H$ take exponentially long to relax. Indeed, the above scaling can be achieved only by effectively having $\left|f_{\lambda}(\mu)\right|^{2} \sim 2^{L}$. Yet, the total spectral weight $\int d \omega\left|f_{\lambda}(\omega)\right|^{2}$ is only polynomially large in the system size, implying that the corresponding spectral weight must be localized in a region $\Delta \omega \sim 2^{-L}$. Combined with expression (8), the latter implies $\partial_{\lambda} H(t)$ takes exponentially long to relax to equilibrium.

For interacting integrable models, we find $\|\mathcal{A}\|^{2} \sim L^{\beta}$, where the exponent $\beta$ is nonuniversal. Since the norm is not exponential in system size, the function $\left|f_{\lambda}(\mu)\right|^{2} \sim 2^{-L}$. This result means that the function should vanish in the zero-frequency limit, which implies oscillatory dynamics of the observable $\partial_{\lambda} H(t)$.

\section{APPENDIX D: EFFECTS OF THE ANISOTROPY IN THE $X X Z$ MODEL}

In this Appendix, we again consider the $X X Z$ Hamiltonian [Eq. (12)]:

$$
H_{X X Z}=\sum_{i=1}^{L-1}\left(\sigma_{i+1}^{x} \sigma_{i}^{x}+\sigma_{i+1}^{y} \sigma_{i}^{y}\right)+\Delta \sum_{i=1}^{L-1} \sigma_{i+1}^{z} \sigma_{i}^{z},
$$

where $\Delta$ is the anisotropy and we take $\Delta=\lambda$ as the adiabatic deformation, but now at different values of $\Delta$. We find that the slope of the AGP norm depends nontrivially on $\Delta$ (Fig. 10). 


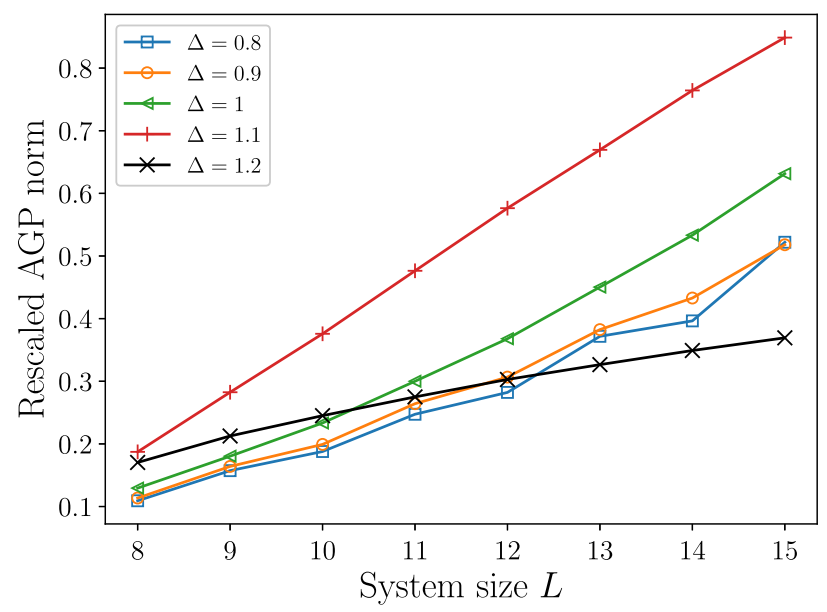

FIG. 10. Anisotropy. Rescaled AGP norm $\left\|\mathcal{A}_{\Delta}\right\|^{2} / L$ for the $X X Z$ chain at different values of the anisotropy $\Delta$.

\section{APPENDIX E: NNN INTERACTIONS IN THE $X X Z$ CHAIN}

In the main text, we study the effect of a strictly local integrability-breaking operator (whose support is a single site). Looking into the effects of the locality, we here study an extensive integrability-breaking operator. We add a nextnearest-neighbor (NNN) interaction to the $X X Z$ chain, with the Hamiltonian given as

$$
H_{\mathrm{NNN}}=H_{X X Z}+\Delta_{2} \sum_{i=1}^{L-2} \sigma_{i+2}^{z} \sigma_{i}^{z} .
$$

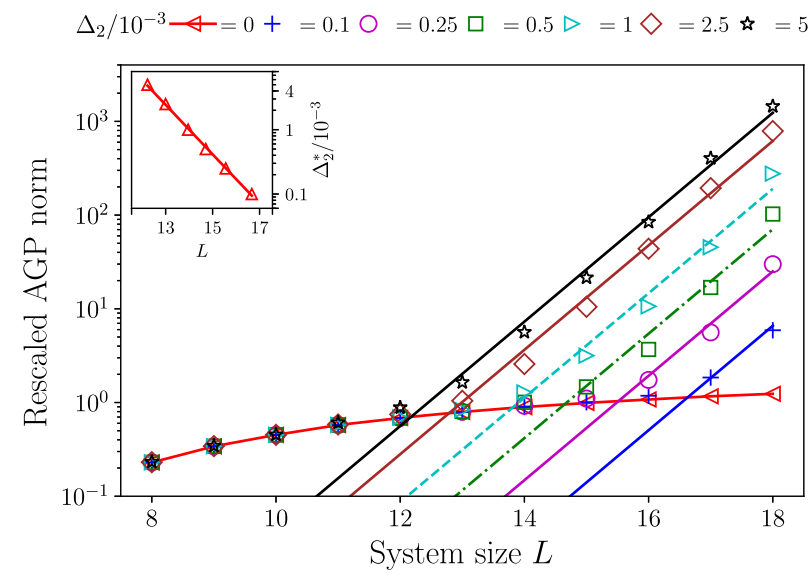

FIG. 11. Integrability breaking through NNN interaction: Rescaled AGP norm $\left\|\mathcal{A}_{\lambda}\right\|^{2} / L$ with $\lambda=\Delta$ of the $X X Z$ chain at $\Delta=1.1$ shows a sharp crossover from polynomial to exponential scaling with the system size, even for very small perturbation strengths $\Delta_{2}$. As $\Delta_{2}$ decreases, the system size where this crossover happens increases. Straight lines are the exponential fits with $\left.\left|A_{\lambda}\right|\right|^{2} / L \sim e^{1.28 L}$. Inset: The integrability-breaking defect energy scales exponentially with the system size, i.e., $\Delta_{2}^{*} \sim e^{-0.9 L}$. This result is calculated for the symmetry sector with zero magnetization.

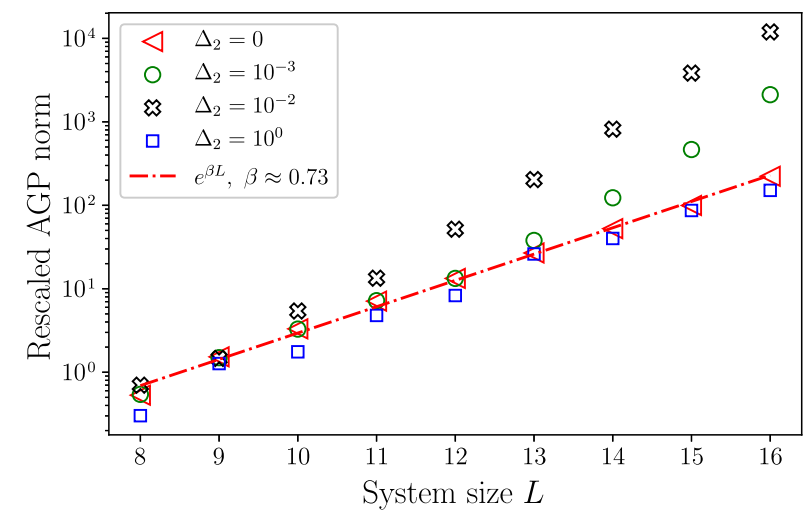

FIG. 12. Integrability-breaking deformation. Rescaled AGP norm $\left\|\mathcal{A}_{\lambda}\right\|^{2} / L$ for the $X X Z$ chain at $\Delta=1.1$ with $\lambda=\Delta_{2}$. This result is calculated for the full Hilbert space, not in any specific symmetry sector.

The above model is chaotic for large enough $\Delta_{2}$ [32]. We choose $\lambda=\Delta$ (Fig. 11) and $\lambda=\Delta_{2}$ (Fig. 12). In the limit $\Delta_{2} \rightarrow 0$, when the above Hamiltonian [Eq. (E1)] is integrable, the former (latter) is the integrability-preserving (breaking) direction. As shown in Figs. 11 and 12, results are similar as for the strictly local perturbation studied in the main text. This similarity implies our results are robust to the nature of the adiabatic deformation.

\section{APPENDIX F: UNIVERSAL SLOPE OF THE AGP NORM}

Here, we study the AGP norm in the $X X Z$ chain in the limit when the magnitude of the integrability-breaking

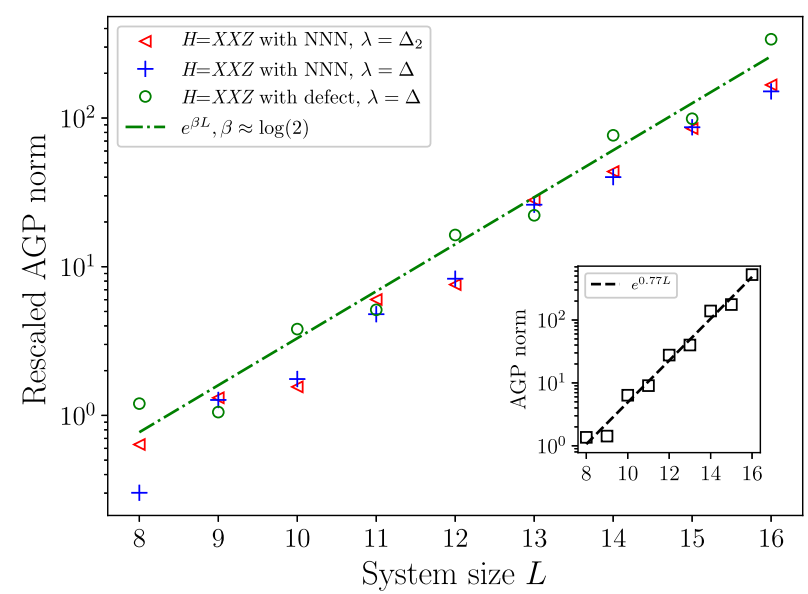

FIG. 13. Universal slope at large integrability-breaking strengths. Rescaled AGP norm $\left\|A_{\lambda}\right\|^{2} / L$ for different models: (A) Model: $X X Z$ chain with NNN interaction [Eq. (E1)]. (a) $\lambda=\Delta$ and (b) $\lambda=\Delta_{2}$. Parameters: $\Delta=1.1$ and $\Delta_{2}=1$. (B) Model: $X X Z$ with a defect in the middle [Eq. (15)]. (a) $\lambda$ is chosen as $\Delta$. Inset: AGP norm $\left\|A_{\lambda}\right\|^{2}$ for $X X Z$ with a defect in the middle model, where $\lambda$ is chosen as $\epsilon_{d}$. Parameters: $\epsilon_{d}=1$ and $\Delta=1.1$. This result is calculated for the full Hilbert space, not in any specific symmetry sector. 
perturbation (either the local defect energy $\epsilon_{d}$ or the NNN interaction strength $\Delta_{2}$ ) is of the same magnitude as the $\Delta / J$ energy scale. In this limit, we find that the AGP has an exponential scaling with the system size characterized by an almost universal slope $\beta \approx \log 2$, which is close to the one predicted by the ETH. Details about the model and its parameters are given in the caption of Fig. 13.

\section{APPENDIX G: COMPARISON TO MANY-BODY "THOULESS CONDUCTANCE"}

For the sake of completeness, we explicitly compare the current results for a single impurity in an $X X Z$ chain to what would have been obtained using previous related measures [59,60]. The authors of Ref. [59] define a manybody Thouless conductance

$$
G_{\mathrm{NN}}=\frac{1}{\mathcal{D}-1} \sum_{n} \log \frac{\left|\left\langle n\left|\partial_{\lambda} H\right| n+1\right\rangle\right|}{E_{n+1}-E_{n}},
$$

denoted as $\mathcal{G}$ in Ref. [59]. The link to the present $\log \left\|\mathcal{A}_{\lambda}\right\|$ is directly apparent. Apart from looking at the typical instead of the mean, the main difference is that $G_{\mathrm{NN}}$ takes into account only transitions to neighboring levels. Numerical results are shown in Fig. 14. In the parameter regime that is studied in this work, the measure behaves as

$$
G_{\mathrm{NN}}=\kappa L+c\left(\epsilon_{d}\right)
$$

where $\kappa \approx 0.87$ is independent of the perturbation $\epsilon_{d}$ and the constant $c$ decreases with $\epsilon_{d}$. A slightly different measure is introduced in Ref. [60], namely,

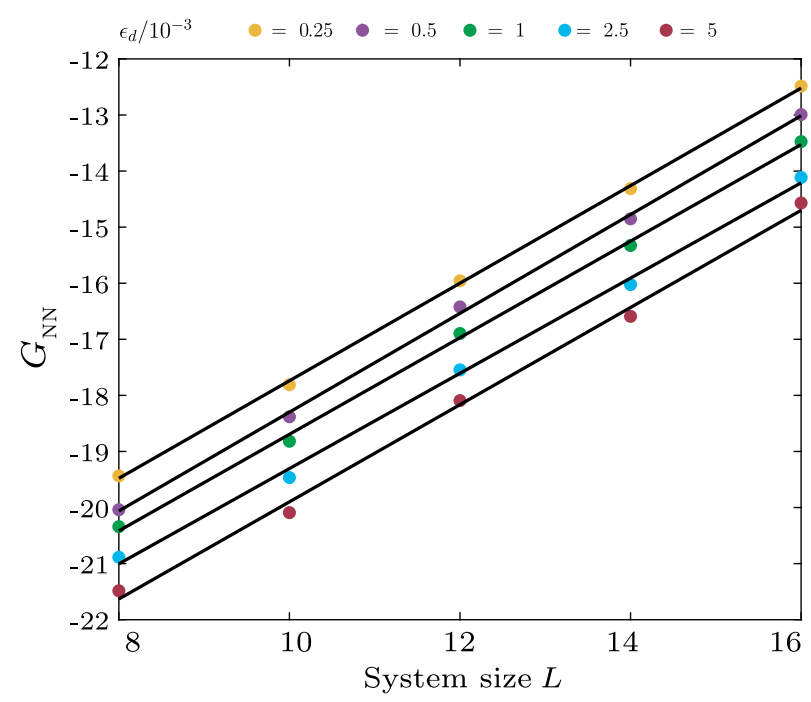

FIG. 14. Probing neighboring levels. $G_{\mathrm{NN}}$ is shown as a function of the system size for various strengths of the integrability-breaking perturbation. All parameters are the same as those discussed in Fig. 3(a). The black lines are linear least square fits to the data.

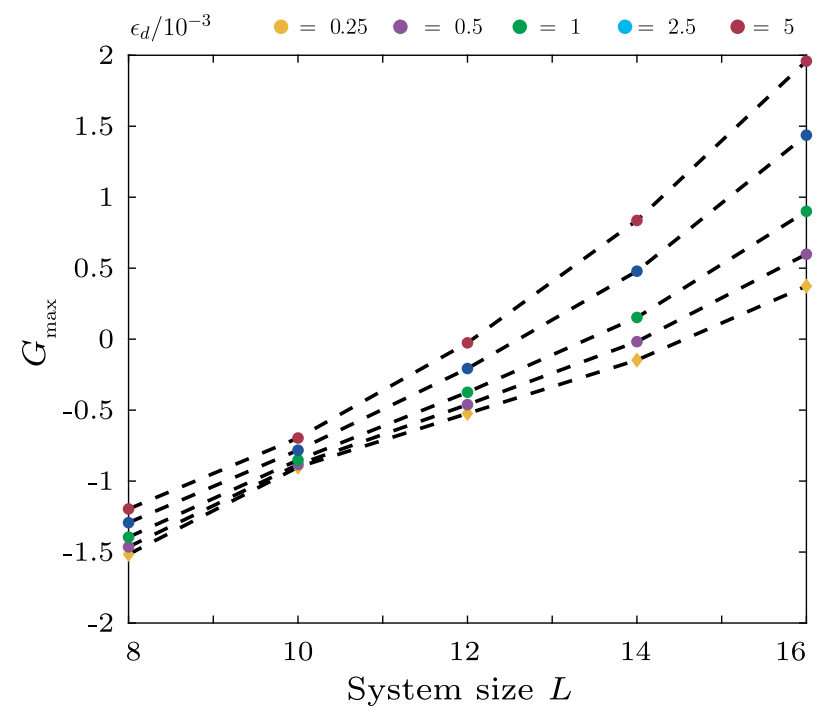

FIG. 15. Probing strongest coupling. $G_{\max }$ is shown as a function of the system size for various strengths of the integrabilitybreaking perturbation. All parameters are the same as those discussed in Fig. 3(a).

$$
G_{\max }=\frac{1}{\mathcal{D}} \sum_{n} \max _{m} \log \frac{\left|\left\langle n\left|\partial_{\lambda} H\right| m\right\rangle\right|}{\left|E_{m}-E_{n}\right|}
$$

denoted as $[v]$ in the original work. The latter removes the bias toward neighboring levels and instead takes the maximum possible ratio of the matrix element to the energy difference. As discussed in the main text, at the onset of chaos, we see a large amount of spectral weight appear at low frequency, and the latter dominates the scaling of the AGP. As a consequence, we expect $G_{\max }$ to be more sensitive to this phenomenon, and this measure should more closely resemble $\log \left\|\mathcal{A}_{\lambda}\right\|$. Numerical results are shown in Fig. 15; these indeed confirm this situation is the case albeit features being less pronounced.

[1] F. Haake, S. Gnutzmann, and M. Kuś, Quantum Signatures of Chaos (Springer, New York, 2013).

[2] H.-J. Stöckmann, Quantum Chaos: An Introduction (Cambridge University Press, Cambridge, England, 1999).

[3] M. Berry, Quantum Chaology, Not Quantum Chaos, Phys. Scr. 40, 335 (1989).

[4] S. H. Shenker and D. Stanford, Black Holes and the Butterfly Effect, J. High Energy Phys. 03 (2014) 067.

[5] J. Maldacena, S. H. Shenker, and D. Stanford, A Bound on Chaos, J. High Energy Phys. 08 (2016) 106.

[6] C. W. von Keyserlingk, T. Rakovszky, F. Pollmann, and S. L. Sondhi, Operator Hydrodynamics, OTOCs, and Entanglement Growth in Systems without Conservation Laws, Phys. Rev. X 8, 021013 (2018).

[7] T. Rakovszky, F. Pollmann, and C. W. von Keyserlingk, Diffusive Hydrodynamics of Out-of-Time-Ordered Correlators with Charge Conservation, Phys. Rev. X 8, 031058 (2018). 
[8] A. Nahum, S. Vijay, and J. Haah, Operator Spreading in Random Unitary Circuits, Phys. Rev. X 8, 021014 (2018).

[9] B. Swingle, Unscrambling the Physics of Out-of-TimeOrder Correlators, Nat. Phys. 14, 988 (2018).

[10] V. Khemani, A. Vishwanath, and D. A. Huse, Operator Spreading and the Emergence of Dissipative Hydrodynamics under Unitary Evolution with Conservation Laws, Phys. Rev. X 8, 031057 (2018).

[11] J. Kudler-Flam, L. Nie, and S. Ryu, Conformal Field Theory and the Web of Quantum Chaos Diagnostics, J. High Energy Phys. 01 (2020) 175.

[12] M. Cencini, F. Cecconi, and A. Vulpiani, Chaos: From Simple Models to Complex Systems (World Scientific, Singapore, 2010), Vol. 17.

[13] J.-S. Caux and J. Mossel, Remarks on the Notion of Quantum Integrability, J. Stat. Mech. (2011) P02023.

[14] E. A. Yuzbashyan and B. S. Shastry, Quantum Integrability in Systems with Finite Number of Levels, J. Stat. Phys. 150, 704 (2013).

[15] E. A. Yuzbashyan, B. S. Shastry, and J. A. Scaramazza, Rotationally Invariant Ensembles of Integrable Matrices, Phys. Rev. E 93, 052114 (2016).

[16] E. Ilievski, M. Medenjak, T. Prosen, and L. Zadnik, Quasilocal Charges in Integrable Lattice Systems, J. Stat. Mech. (2016) 064008.

[17] T. A. Brody, J. Flores, J. B. French, P. Mello, A. Pandey, and S. S. Wong, Random-Matrix Physics: Spectrum and Strength Fluctuations, Rev. Mod. Phys. 53, 385 (1981).

[18] T. Guhr, A. Müller-Groeling, and H. A. Weidenmüller, Random-Matrix Theories in Quantum Physics: Common Concepts, Phys. Rep. 299, 189 (1998).

[19] M. L. Mehta, Random Matrices, 3rd ed. (Elsevier, New York, 2004).

[20] E. P. Wigner, On the Distribution of the Roots of Certain Symmetric Matrices, Ann. Math. 67, 325 (1958).

[21] E. P. Wigner, Characteristic Vectors of Bordered Matrices with Infinite Dimensions II, in The Collected Works of Eugene Paul Wigner (Springer, New York, 1993), pp. 541-545.

[22] O. Bohigas, M.-J. Giannoni, and C. Schmit, Characterization of Chaotic Quantum Spectra and Universality of Level Fluctuation Laws, Phys. Rev. Lett. 52, 1 (1984).

[23] M. V. Berry and M. Tabor, Level Clustering in the Regular Spectrum, Proc. R. Soc. A 356, 375 (1977).

[24] J. M. Deutsch, Quantum Statistical Mechanics in a Closed System, Phys. Rev. A 43, 2046 (1991).

[25] M. Srednicki, Chaos and Quantum Thermalization, Phys. Rev. E 50, 888 (1994).

[26] M. Rigol, V. Dunjko, and M. Olshanii, Thermalization and Its Mechanism for Generic Isolated Quantum Systems, Nature (London) 452, 854 (2008).

[27] F. Borgonovi, F. M. Izrailev, L. F. Santos, and V. G. Zelevinsky, Quantum Chaos and Thermalization in Isolated Systems of Interacting Particles, Phys. Rep. 626, 1 (2016).

[28] L. D'Alessio, Y. Kafri, A. Polkovnikov, and M. Rigol, From Quantum Chaos and Eigenstate Thermalization to Statistical Mechanics and Thermodynamics, Adv. Phys. 65, 239 (2016).

[29] J. M. Deutsch, Eigenstate Thermalization Hypothesis, Rep. Prog. Phys. 81, 082001 (2018).
[30] S. Müller, S. Heusler, P. Braun, F. Haake, and A. Altland, Semiclassical Foundation of Universality in Quantum Chaos, Phys. Rev. Lett. 93, 014103 (2004).

[31] B. Bertini, P. Kos, and T. Prosen, Exact Spectral Form Factor in a Minimal Model of Many-Body Quantum Chaos, Phys. Rev. Lett. 121, 264101 (2018).

[32] A. Gubin and L. F. Santos, Quantum Chaos: An Introduction via Chains of Interacting Spins $1 / 2$, Am. J. Phys. 80, 246 (2012).

[33] K. Kudo and T. Deguchi, Unexpected Non-Wigner Behavior in Level-Spacing Distributions of Next-Nearest-Neighbor Coupled XXZ Spin Chains, Phys. Rev. B 68, 052510 (2003).

[34] J. M. G. Gómez, R. A. Molina, A. Relano, and J. Retamosa, Misleading Signatures of Quantum Chaos, Phys. Rev. E 66, 036209 (2002).

[35] V. Oganesyan and D. A. Huse, Localization of Interacting Fermions at High Temperature, Phys. Rev. B 75, 155111 (2007).

[36] Y. Y. Atas, E. Bogomolny, O. Giraud, and G. Roux, Distribution of the Ratio of Consecutive Level Spacings in Random Matrix Ensembles, Phys. Rev. Lett. 110, 084101 (2013).

[37] E. J. Torres-Herrera and L. F. Santos, Dynamical Manifestations of Quantum Chaos: Correlation Hole and Bulge, Phil. Trans. R. Soc. A 375, 20160434 (2017).

[38] M. Schiulaz, E. J. Torres-Herrera, and L. F. Santos, Thouless and Relaxation Time Scales in Many-Body Quantum Systems, Phys. Rev. B 99, 174313 (2019).

[39] M. Kolodrubetz, D. Sels, P. Mehta, and A. Polkovnikov, Geometry and Non-adiabatic Response in Quantum and Classical Systems, Phys. Rep. 697, 1 (2017).

[40] D. N. Page, Geometrical Description of Berry's Phase, Phys. Rev. A 36, 3479 (1987).

[41] S. Kobayashi and K. Nomizu, Foundations of Differential Geometry (Wiley, New York, 1963), Vol. 1.

[42] J. Provost and G. Vallee, Riemannian Structure on Manifolds of Quantum States, Commun. Math. Phys. 76, 289 (1980).

[43] M. Berry, Transitionless Quantum Driving, J. Phys. A 42, 365303 (2009).

[44] M. Demirplak and S. A. Rice, Assisted Adiabatic Passage Revisited, J. Phys. Chem. B 109, 6838 (2005).

[45] M. Demirplak and S. A. Rice, Adiabatic Population Transfer with Control Fields, J. Phys. Chem. A 107, 9937 (2003).

[46] J. Šuntajs, J. Bonča, T. Prosen, and L. Vidmar, Quantum Chaos Challenges Many-Body Localization, arXiv:1905 .06345 .

[47] R. Modak and S. Mukerjee, Finite Size Scaling in Crossover among Different Random Matrix Ensembles in Microscopic Lattice Models, New J. Phys. 16, 093016 (2014).

[48] R. Modak, S. Mukerjee, and S. Ramaswamy, Universal Power Law in Crossover from Integrability to Quantum Chaos, Phys. Rev. B 90, 075152 (2014).

[49] G. Gallavotti, The Fermi-Pasta-Ulam Problem: A Status Report (Springer, New York, 2007), Vol. 728.

[50] C. Danieli, D. Campbell, and S. Flach, Intermittent ManyBody Dynamics at Equilibrium, Phys. Rev. E 95, 060202(R) (2017). 
[51] S. D. Pace and D. K. Campbell, Behavior and Breakdown of Higher-Order Fermi-Pasta-Ulam-Tsingou Recurrences, Chaos 29, 023132 (2019).

[52] J. Eisert, M. Friesdorf, and C. Gogolin, Quantum Many-Body Systems out of Equilibrium, Nat. Phys. 11, 124 (2015).

[53] B. Swingle, Unscrambling the Physics of Out-of-TimeOrder Correlators, Nat. Phys. 14, 988 (2018).

[54] R. Lewis-Swan, A. Safavi-Naini, A. Kaufman, and A. Rey, Dynamics of Quantum Information, Nat. Rev. Phys. 1, 627 (2019).

[55] T. Zhou and D. J. Luitz, Operator Entanglement Entropy of the Time Evolution Operator in Chaotic Systems, Phys. Rev. B 95, 094206 (2017).

[56] V. Alba, J. Dubail, and M. Medenjak, Operator Entanglement in Interacting Integrable Quantum Systems: The Case of the Rule 54 Chain, Phys. Rev. Lett. 122, 250603 (2019).

[57] D. E. Parker, X. Cao, A. Avdoshkin, T. Scaffidi, and E. Altman, A Universal Operator Growth Hypothesis, Phys. Rev. X 9, 041017 (2019).

[58] A. Avdoshkin and A. Dymarsky, Euclidean Operator Growth and Quantum Chaos, arXiv:1911.09672 [Phys. Rev. Research (to be published)].

[59] M. Serbyn, Z. Papić, and D. A. Abanin, Criterion for ManyBody Localization-Delocalization Phase Transition, Phys. Rev. X 5, 041047 (2015).

[60] P. J. Crowley and A. Chandran, Avalanche Induced Coexisting Localised and Thermal Regions in Disordered Chains, Phys. Rev. Research 2, 033262 (2020).

[61] T. LeBlond, K. Mallayya, L. Vidmar, and M. Rigol, Entanglement and Matrix Elements of Observables in Interacting Integrable Systems, Phys. Rev. E 100, 062134 (2019).

[62] R. Mondaini and M. Rigol, Eigenstate Thermalization in the Two-Dimensional Transverse Field Ising Model. II. OffDiagonal Matrix Elements of Observables, Phys. Rev. E 96, 012157 (2017).

[63] M. Brenes, T. LeBlond, J. Goold, and M. Rigol, Eigenstate Thermalization in a Locally Perturbed Integrable System, Phys. Rev. Lett. 125, 070605 (2020).

[64] M. Brenes, J. Goold, and M. Rigol, Ballistic vs Diffusive Low-Frequency Scaling in the XXZ and a Locally Perturbed XXZ Chain, Phys. Rev. B 102, 075127 (2020).

[65] M. Berry and J. M. Robbins, Chaotic Classical and HalfClassical Adiabatic Reactions: Geometric Magnetism and Deterministic Friction, Proc. R. Soc. A 442, 659 (1993).

[66] C. Jarzynski, Geometric Phases and Anholonomy for a Class of Chaotic Classical Systems, Phys. Rev. Lett. 74, 1732 (1995).

[67] P. W. Claeys, M. Pandey, D. Sels, and A. Polkovnikov, Floquet-Engineering Counterdiabatic Protocols in Quantum Many-Body Systems, Phys. Rev. Lett. 123, 090602 (2019).

[68] M. Hastings, Quasi-Adiabatic Continuation for Disordered Systems: Applications to Correlations, Lieb-Schultz-Mattis, and Hall Conductance, arXiv:1001.5280.

[69] S. Bachmann, S. Michalakis, B. Nachtergaele, and R. Sims, Automorphic Equivalence within Gapped Phases of
Quantum Lattice Systems, Commun. Math. Phys. 309, 835 (2012).

[70] S. Bachmann, W. De Roeck, and M. Fraas, Adiabatic Theorem for Quantum Spin Systems, Phys. Rev. Lett. 119, 060201 (2017).

[71] R. Orbach, Linear Antiferromagnetic Chain with Anisotropic Coupling, Phys. Rev. 112, 309 (1958).

[72] C. N. Yang and C.P. Yang, One-Dimensional Chain of Anisotropic Spin-Spin Interactions. I. Proof of Bethe's Hypothesis for Ground State in a Finite System, Phys. Rev. 150, 321 (1966).

[73] B. Sutherland, Beautiful Models: 70 Years of Exactly Solved Quantum Many-Body Problems (World Scientific, Singapore, 2004).

[74] M. Gaudin, The Bethe Wavefunction (Cambridge University Press, Cambridge, England, 2014), translated by JeanSébastien Caux.

[75] F. Franchini, An Introduction to Integrable Techniques for One-Dimensional Quantum Systems (Springer, New York, 2017), Vol. 940.

[76] S. Sachdev, Quantum Phase Transitions (Wiley Online Library, New York, 2007).

[77] A. del Campo, M. M. Rams, and W. H. Zurek, Assisted Finite-Rate Adiabatic Passage across a Quantum Critical Point: Exact Solution for the Quantum Ising Model, Phys. Rev. Lett. 109, 115703 (2012).

[78] H. Kim and D. A. Huse, Ballistic Spreading of Entanglement in a Diffusive Nonintegrable System, Phys. Rev. Lett. 111, 127205 (2013).

[79] We divide the norm of the AGP by the system size for extensive perturbations, to account for the trivial extensivity of the AGP.

[80] L. F. Santos, Integrability of a Disordered Heisenberg Spin1/2 Chain, J. Phys. A 37, 4723 (2004).

[81] E. J. Torres-Herrera and L. F. Santos, Local Quenches with Global Effects in Interacting Quantum Systems, Phys. Rev. E 89, 062110 (2014).

[82] J. Möser, On Invariant Curves of Area-Preserving Mappings of an Annulus, Matematika 6, 51 (1962), http://www .mathnet.ru/php/archive.phtml?wshow=paper\&jrnid= mat\&paperid=236\&option_lang=eng.

[83] A. N. Kolmogorov, On Conservation of Conditionally Periodic Motions for a Small Change in Hamilton's Function, Dokl. Akad. Nauk SSSR 98, 527 (1954).

[84] V. I. Arnold, Proof of a Theorem of A. N. Kolmogorov on the Invariance of Quasi-Periodic Motions Under Small Perturbations of the Hamiltonian, Russ. Math. Surv. 18, 9 (1963).

[85] D. A. Rabson, B. N. Narozhny, and A. J. Millis, Crossover from Poisson to Wigner-Dyson Level Statistics in Spin Chains with Integrability Breaking, Phys. Rev. B 69, 054403 (2004).

[86] L. F. Santos and M. Rigol, Onset of Quantum Chaos in OneDimensional Bosonic and Fermionic Systems and Its Relation to Thermalization, Phys. Rev. E 81, 036206 (2010).

[87] R. Nandkishore and D. A. Huse, Many-Body Localization and Thermalization in Quantum Statistical Mechanics, Annu. Rev. Condens. Matter Phys. 6, 15 (2015). 
[88] D. A. Abanin and Z. Papić, Recent Progress in Many-Body Localization, Ann. Phys. (Berlin) 529, 1700169 (2017).

[89] N. Chavda, H. Deota, and V. Kota, Poisson to GOE Transition in the Distribution of the Ratio of Consecutive Level Spacings, Phys. Lett. A 378, 3012 (2014).

[90] R. E. Prange, The Spectral Form Factor Is Not SelfAveraging, Phys. Rev. Lett. 78, 2280 (1997).

[91] P. Braun and F. Haake, Self-Averaging Characteristics of Spectral Fluctuations, J. Phys. A 48, 135101 (2015).

[92] T. Villazon, P. W. Claeys, M. Pandey, A. Polkovnikov, and A. Chandran, Persistent Dark States in Anisotropic Central Spin Models, arXiv:2005.13556.

[93] B. Bertini, P. Kos, and T. Prosen, Operator Entanglement in Local Quantum Circuits I: Chaotic Dual-Unitary Circuits, SciPost Phys. 8, 67 (2020).
[94] A. Dymarsky and H. Liu, New Characteristic of Quantum Many-Body Chaotic Systems, Phys. Rev. E 99, 010102(R) (2019).

[95] P. Sierant, A. Maksymov, M. Kuś, and J. Zakrzewski, Fidelity Susceptibility in Gaussian Random Ensembles, Phys. Rev. E 99, 050102(R) (2019).

[96] P. Weinberg and M. Bukov, Quspin: A Python Package for Dynamics and Exact Diagonalisation of Quantum Many Body Systems Part I: Spin Chains, SciPost Phys. 2, 003 (2017).

[97] P. Weinberg and M. Bukov, Quspin: A Python Package for Dynamics and Exact Diagonalisation of Quantum Many Body Systems. Part II: Bosons, Fermions and Higher Spins, SciPost Phys. 7, 020 (2019). 